\title{
Resveratrol alleviates diabetic cardiomyopathy in rats by improving mitochondrial function through PGC-1 $\alpha$ deacetylation
}

\author{
Wei-jin FANG", Chun-jiang WANG\#, Yang HE, Yu-lu ZHOU, Xiang-dong PENG, Shi-kun LIU* \\ Department of Pharmacy, the Third Xiangya Hospital of Central South University, Changsha 410013, China
}

\begin{abstract}
Recent evidence shows that resveratrol (RSV) may ameliorate high-glucose-induced cardiac oxidative stress, mitochondrial dysfunction and myocardial fibrosis in diabetes. However, the mechanisms by which RSV regulates mitochondrial function in diabetic cardiomyopathy have not been fully elucidated. Mitochondrial dysfunction contributes to cardiac dysfunction in diabetic patients, which is associated with dysregulation of peroxisome proliferator-activated receptor gamma coactivator-1 $\alpha$ (PGC-1 $\alpha)$. In this study we examined whether resveratrol alleviated cardiac dysfunction in diabetes by improving mitochondrial function via SIRT1-mediated PGC-1 $\alpha$ deacetylation. T2DM was induced in rats by a high-fat diet combined with STZ injection. Diabetic rats were orally administered RSV (50 $\mathrm{mg} \cdot \mathrm{kg}^{-1} \cdot \mathrm{d}^{-1}$ ) for 16 weeks. RSV administration significantly attenuated diabetes-induced cardiac dysfunction and hypertrophy evidenced by increasing ejection fraction (EF\%), fraction shortening (FS\%), ratio of early diastolic peak velocity (E velocity) and late diastolic peak velocity (A velocity) of the LV inflow (E/A ratio) and reducing expression levels of pro-hypertrophic markers ANP, BNP and $\beta$-MHC. Furthermore, manganese superoxide dismutase (SOD) activity, ATP content, mitochondrial DNA copy number, mitochondrial membrane potential and the expression of nuclear respiration factor (NRF) were all significantly increased in diabetic hearts by RSV administration, whereas the levels of malondialdehvde (MDA) and uncoupling protein 2 (UCP2) were significantly decreased. Moreover, RSV administration significantly activated SIRT1 expression and increased PGC-1 $\alpha$ deacetylation. H9c2 cells cultured in a high glucose ( $\mathrm{HG}, 30 \mathrm{mmol} / \mathrm{L}$ ) condition were used for further analyzing the role of SIRT1/PGC-1 $\alpha$ pathway in RSV regulation of mitochondrial function. RSV ( $20 \mu \mathrm{mol} / \mathrm{L})$ caused similar beneficial effects in HG-treated H9c2 cells in vitro as in diabetic rats, but these protective effects were abolished by addition of a SIRT1 inhibitor sirtinol ( $25 \mu \mathrm{mol} / \mathrm{L})$ or by SIRT1 siRNA transfection. In H9c2 cells, RSV-induced PGC-1 $\alpha$ deacetylation was dependent on SIRT1, which was also abolished by a SIRT1 inhibitor and SIRT1 siRNA transfection. Our results demonstrate that resveratrol attenuates cardiac injury in diabetic rats through regulation of mitochondrial function, which is mediated partly through SIRT1 activation and increased PGC-1 $\alpha$ deacetylation.
\end{abstract}

Keywords: resveratrol; diabetic cardiomyopathy; mitochondrial dysfunction; oxidative stress; SIRT1; PGC-1 $\alpha$; sirtinol

Acta Pharmacologica Sinica (2018) 39: 59-73; doi: 10.1038/aps.2017.50; published online 3 Aug 2017

\section{Introduction}

Identification of new and improved treatment modalities for diabetes mellitus is urgently needed given its growing incidence and increased mortality due to cardiovascular complications $^{[1]}$. Among these complications, diabetic cardiomyopathy (DCM) develops in both type 1 and type 2 diabetes and is characterized by diastolic dysfunction in the early stage and systolic dysfunction in the advanced stage. DCM contributes to the increased morbidity and mortality of heart failure in diabetic mellitus ${ }^{[2]}$.

\footnotetext{
\# These authors contributed equally to this work.

* To whom correspondence should be addressed.

E-mail L8618496@126.com

Received 2016-12-29 Accepted 2017-03-28
}

Excess reactive oxygen species (ROS) production leading to oxidative stress has been widely implicated in both the onset of diabetes and many of its complications ${ }^{[3]}$. Mitochondria are major sites of superoxide radical generation, which is produced as a by-product of adenosine triphosphate (ATP) formation by oxidative phosphorylation. Mitochondrial antioxidative enzymes, such as manganese SOD (MnSOD), can detoxify ROS. However, excess ROS generation can overwhelm these defense mechanisms and lead to mitochondrial damage. Mitochondria themselves, and in particular mitochondrial DNA (mtDNA), are susceptible to ROS, which may be regulated by peroxisome proliferator-activated receptor gamma coactivator-1a (PGC-1a) and are vulnerable to oxidative damage $^{[4]}$. Growing evidence has indicated that diabetes impairs 
heart mitochondrial function and contributes to $\mathrm{DCM}^{[5]}$; thus, preventing cardiac oxidative stress and mitochondrial dysfunction in diabetic patients can improve cardiac function and reduce hypertrophy ${ }^{[6]}$. Restoration of mitochondrial function also alleviates diabetic cardiomyopathy associated with contractile dysfunction in $d b / d b$ mice $^{[7]}$, suggesting that mitochondrial dysfunction and related oxidative stress are major pathological factors in diabetic complications. However, the mechanisms underlying these processes have not been investigated in diabetic cardiomyopathy.

Resveratrol (RSV) is a polyphenolic flavonoid with pleotropic activities that exerts a wide variety of beneficial effects in a SIRT1-dependent manner to slow or prevent the development of many illnesses, including diabetes and cardiovascular diseases ${ }^{[8,9]}$. Zhou et al found that RSV regulates mitochondrial reactive oxygen species homeostasis in human vascular endothelial cells via increased SOD2 activity and enhanced PGC-1a expression ${ }^{[10]}$. Recent studies have suggested that RSV may ameliorate high-glucose-induced cardiac oxidative stress, mitochondrial dysfunction and myocardial fibrosis in diabetes ${ }^{[6,11-13]}$. However, the mechanisms by which RSV regulates mitochondrial function in diabetic cardiomyopathy have not been fully elucidated.

In the present study, we hypothesized that RSV has a protective effect by improving mitochondrial dysfunction in diabetic cardiomyopathy through SIRT1-mediated PGC-1a deacetylation. We investigated the effect of resveratrol on mitochondrial function and oxidative stress in the hearts of diabetic rats induced by streptozotocin (STZ) combined with a high-fat diet and in $\mathrm{H} 9 \mathrm{c} 2$ cells cultured in high-glucose conditions. The role of the SIRT1/PGC-1a pathway in resveratrolmediated regulation of mitochondrial function was identified using in vitro experiments.

\section{Materials and methods Reagents}

Streptozotocin (STZ), insulin, resveratrol (RSV) and sirtinol were purchased from Sigma-Aldrich (St Louis, MO, USA). Serum triglyceride (TG), total cholesterol (TC), low-density lipoprotein cholesterol (LDL-C), and high-density lipoprotein cholesterol (HDL-C) assay kits were obtained from Dongou Co, LTD (Wenzhou, China). The glycosylated hemoglobin assay kit was obtained from Jiancheng Bioengineering Institute (Nanjing, China). RIPA lysis buffer, the BCA protein assay kit, the BeyoECL Plus kit, JC-1 and 2',7'-dichloro-fluorescin diacetate (DCFH-DA) were acquired from Beyotime Biotechnology (Shanghai, China). The primers for atrial natriuretic peptide (ANP), brain natriuretic peptide (BNP), $\beta$-myosin heavy chain $(\beta-\mathrm{MHC})$, PGC-1 $\alpha$, nuclear respiratory factor (NRF), uncoupling protein 2 (UCP2) and cytochrome $c$ oxidase 1 (COX 1) were synthesized by Invitrogen Life Technologies (Carlsbad, NY, USA). The reverse-transcription assay kit was obtained from TaKaRa (Shiga, Japan).

\section{Antibodies}

The antibodies used for Western blot and immunoprecipita- tion were as follows: anti-PGC-1a, anti-SIRT1, anti-UCP2 and anti-glyceraldehyde-3-phosphate dehydrogenase (GAPDH) antibodies were obtained from Santa Cruz (Texas, USA); antiAkt, anti-phosphor-Akt (Ser473), and anti-acetylated-lysine antibodies were obtained from Cell Signaling Technology (Massachusetts, USA).

\section{Animals}

All animal experiments were performed according to the Guidelines of Animal Experiments from the Committee of Medical Ethics at the National Health Department of China and were approved by Central South University. Specific pathogen-free (SPF) male SD rats, weighing $150 \pm 20 \mathrm{~g}$ at 8 weeks of age, were obtained from the Center of Laboratory Animal Science of Guangdong (Guangzhou, China). Animals were housed in plastic cages with well-ventilated stainless steel grid tops at $22^{\circ} \mathrm{C}$ with a $12-\mathrm{h}$ light/dark cycle ${ }^{[14]}$. Upon arrival, the rats were acclimatized for one week and fed normal chow ad libitum. A model of type 2 diabetes mellitus (T2DM) was established by feeding the rats a high-fat diet (60\% chow, $10 \%$ egg yolk powder, $10 \%$ lard, $1.5 \%$ cholesterol and $0.1 \%$ sodium cholate) and injecting them with low-dose STZ $(35 \mathrm{mg} / \mathrm{kg}$ intraperitoneally in a $0.1 \mathrm{~mol} / \mathrm{L}$ citrate buffer, $\mathrm{pH} 4.5)$ as reported previously ${ }^{[15]}$. The control groups received normal chow and the same volume of citrate buffer. Diabetes onset was confirmed by 2 consecutive observations of blood glucose levels $\geq 16 \mathrm{mmol} / \mathrm{L}$ by random measurement every other day. After confirmation of diabetes, the rats were fed the high-fat diet for an additional 16 weeks. During this period, the diabetic rats were given resveratrol (RSV, $50 \mathrm{mg} / \mathrm{kg}$ ) by oral gavage. Age-matched control rats were fed normal chow and received equivalent volumes of resveratrol by intragastric administration for 16 weeks. At the end of the study, the rats were anesthetized with $10 \%$ chloral hydrate $(30 \mathrm{mg} / \mathrm{kg}$, ip). Plasma $(8-10 \mathrm{~mL})$ was immediately collected from the carotid artery and processed to yield serum. After washing in icecold saline solution, rat hearts were weighed and carefully examined for morphological changes. The remaining cardiac samples were frozen and stored at $-80^{\circ} \mathrm{C}$.

\section{H9c2 cell cultures and treatment}

The H9c2 cell line is a subclone of the original clonal cell line derived from embryonic BD1X rat heart tissue and therefore was used to mimic cardiac myocytes to explore various underlying mechanisms in the present study. H9c2 cells were obtained from the Institute of Biochemistry and Cell Biology (Shanghai Institute for Biological Science, the Chinese Academy of Sciences, Shanghai, China) and cultured in Dulbecco's modified Eagle's medium (DMEM, Gibco, Eggenstein, Germany) containing $5.5 \mathrm{mmol} / \mathrm{L} D$-glucose supplemented with $10 \% \mathrm{FBS}, 100 \mathrm{U} / \mathrm{mL}$ penicillin and $100 \mathrm{mg} / \mathrm{mL}$ streptomycin at $37^{\circ} \mathrm{C}$ in a humidified atmosphere of $5 \% \mathrm{CO}_{2}{ }^{[14,16]}$. The cells were divided into the following groups after they reached $70 \%$ confluency: (1) the high-glucose group, in which cardiomyocytes were incubated with DMEM medium containing $30 \mathrm{mmol} / \mathrm{L}$ glucose ${ }^{[17]}$; (2) the RSV+HG group, in which 
$20 \mu \mathrm{mol} / \mathrm{L}$ resveratrol was added to the DMEM medium with $30 \mathrm{mmol} / \mathrm{L}$ glucose; (3) the sirtinol group, in which 25 $\mu \mathrm{mol} / \mathrm{L}$ sirtinol was added to the DMEM medium; (4) the sirtinol+HG group, in which $25 \mu \mathrm{mol} / \mathrm{L}$ sirtinol was added to DMEM medium with $30 \mathrm{mmol} / \mathrm{L}$ glucose; and (5) the $\mathrm{RSV}+$ sirtinol+HG group, in which $20 \mu \mathrm{mol} / \mathrm{L}$ resveratrol and $25 \mu \mathrm{mol} / \mathrm{L}$ sirtinol were added to the DMEM medium with 30 $\mathrm{mmol} / \mathrm{L}$ glucose. All cell cultures were maintained at $37^{\circ} \mathrm{C}$ in a humidified incubator containing $5 \% \mathrm{CO}_{2}$ for $48 \mathrm{~h}$ and then harvested and stored at $-80^{\circ} \mathrm{C}$ for further analysis.

\section{SiRNA transfection}

The H9c2 cells were transfected with siRNA as previously described $^{[18]}$. In brief, H9c2 cells were transfected transiently with SIRT1 or control siRNA (GenePharma, Shanghai, China) using Lipofectamine 2000 (Invitrogen). The sequences of siRNA for SIRT1 were as follows: forward primer, 5'-CACCUGAGUUGGAUGAUAUTT-3' and reverse primer, 5'-AUAUCAUCCAACUAGGUGTT-3'. The cells were incubated with RSV or HG after siRNA transfection.

\section{Echocardiography}

Transthoracic two-dimensional and M-mode echocardiograms were performed using a Vevo 2100 (VisualSonics Inc, Canada) equipped with a $30-\mathrm{MHz}$ transducer at 16 weeks after the diabetic rat model was established ${ }^{[19]}$. The rats were anesthetized with a constant inflow of $3 \%$ isoflurane mixed with pure oxygen. They were placed on a heated platform with embedded ECG contact pads to maintain their body temperatures above $36^{\circ} \mathrm{C}$. Ultrasound gel was placed on the chest after removal of all the hair on the thorax with hair removal solution before the $30 \mathrm{MHz}$ scan head was utilized ${ }^{[20]}$.

Two-dimensionally targeted M-mode echocardiographic images were obtained in both long- and short-axis views at the level of the papillary muscles and recorded at a speed of $66 \mathrm{~mm} / \mathrm{s}$. LV internal diameters and wall thicknesses were obtained from cross-sectional short-axis views. Heart rate was also recorded ${ }^{[21]}$. The shortening fraction (LVFS\%) and ejection fraction (LVEF\%) were calculated from the M-mode tracing to reflect systolic function. Measurements of diastolic dysfunction, such as mitral valve E/A ratios, were made from $2 \mathrm{D}$ parasternal short axis views in diastole $\mathrm{e}^{[22]}$.

\section{Heart weight to body weight $(\mathrm{HW} / \mathrm{BW})$ ratios}

At the end of the experiment, the rats were sacrificed, and the hearts were harvested, dried and weighed. In addition, total body weight (BW) was obtained before initiation of treatment and at the time of sacrifice. Cardiac hypertrophy was detected by determining the heart dry weight to total body weight (HW/BW) ratio. The hearts were then frozen in liquid nitrogen and stored at $-80^{\circ} \mathrm{C}$ until use.

\section{Hematoxylin and eosin (H\&E) staining}

To determine whether diabetes mellitus altered the cardiac morphology, the hearts were harvested at the end of the study. These hearts were photographed and fixed in $4 \%$ para- formaldehyde $\mathrm{e}^{[23]}$. Then, tissues were dehydrated, embedded in paraffin blocks, cut into 5- $\mu \mathrm{m}$ sections, and mounted on 3-aminopropyltriethoxysilane-coated slides. The sections were deparaffinized, rehydrated, washed with $\mathrm{H}_{2} \mathrm{O}$ and stained with hematoxylin-eosin (H\&E) for assessment of myocardial hypertrophy by measuring cardiomyocyte diameter using an Olympus fluorescent microscope (Olympus, Japan) under $\times 200$ magnifications.

\section{Myocardial ATP content assay}

The ATP contents in the heart tissue or H9c2 cells were measured using a bioluminescence method as previously described $^{[24,25]}$ and an ATP-dependent bioluminescence assay kit (\#S0026, Beyotime, China) ${ }^{[26]}$. The assay included luciferase, which generates a stable luminescent signal proportional to the amount of ATP present ${ }^{[27]}$. Briefly, at the end of the experiment, the myocardial tissue homogenate was harvested from $30 \mathrm{mg}$ myocardial tissue and fully lysed by adding 300 $\mu \mathrm{L}$ ATP lysis buffer in a mechanical homogenizer. The cultured $\mathrm{H} 9 \mathrm{c} 2$ cardiomyocytes were collected in $1.5-\mathrm{mL}$ tubes after different treatments. The appropriate volume of lysis solution was added to each tube, and the tubes were centrifuged at $12000 \mathrm{r} / \mathrm{min}$ for $10 \mathrm{~min}$ at $4{ }^{\circ} \mathrm{C}$. The supernatants were collected for further analysis. According to the manufacturer's instructions, $100 \mu \mathrm{L}$ of the sample homogenate was added to $100 \mu \mathrm{L}$ ATP detection working solution and gently mixed. A microplate luminometer (BioTek, USA) was then used to determine the $O D$ of the samples with an integration time of $10 \mathrm{~s}$ per well ${ }^{[28]}$. The standard curve was generated using the same method and concentrations of ATP in myocardial tissue or $\mathrm{H} 9 \mathrm{c} 2$ cells.

\section{Evaluation of mitochondrial membrane potential $(\Delta \psi \mathrm{m})$}

Changes in $\Delta \psi \mathrm{m}$ were detected using a mitochondrial staining kit that uses 5,50,6,60-tetrachloro-1,10,3,30-tetraethylbenzimidazolylcarbocyanine iodide (JC-1) according to the user manual. JC-1 is a fluorescent probe that concentrates in the mitochondrial matrix to form J-aggregates when $\Delta \psi m$ is high, emitting red fluorescence. JC-1 cannot concentrate in the mitochondrial matrix when $\Delta \psi \mathrm{m}$ is low, and the JC- 1 monomer produces green fluorescence. The relative proportion of red and green fluorescence is commonly used to measure the degree of mitochondrial depolarization ${ }^{[29]}$. A decrease in red/green ratio is indicative of a reduced $\Delta \psi \mathrm{m}$. For this assay, H9c2 cells were grown in 96-well plates and treated with or without HG (30 $\mathrm{mmol} / \mathrm{L}), \mathrm{RSV}(20 \mu \mathrm{mol} / \mathrm{L})$ and sirtinol $(25 \mu \mathrm{mol} / \mathrm{L})$. After a $48 \mathrm{~h}$ exposure, the cells were washed with PBS and incubated with $2 \mu \mathrm{mol} / \mathrm{L}$ of JC- 1 dye in DMEM at $37^{\circ} \mathrm{C}$ for $20 \mathrm{~min}$. The images were then taken by a High Content Screening System (PerkinElmer, Massachusetts, USA), and the mitochondrial depolarization patterns of cells were examined using an Operetta ${ }^{\circledR}$ High Content Imaging System (PerkinElmer, Massachusetts, USA).

Determination of intracellular reactive oxygen species (ROS) Intracellular ROS contents were detected utilizing $2^{\prime}, 7^{\prime}$ - 
dichloro-fluorescin diacetate (DCFH-DA) ${ }^{[30]}$. Briefly, $5 \times 10^{5}$ cells were plated on a 96-well plate, allowed to attach overnight, and then treated with or without HG ( $30 \mathrm{mmol} / \mathrm{L})$, RSV $(20 \mu \mathrm{mol} / \mathrm{L})$ and sirtinol $(25 \mu \mathrm{mol} / \mathrm{L})$ for $48 \mathrm{~h}$. Cells were washed with PBS and stained with $40 \mu \mathrm{mol} / \mathrm{L}$ DCFH-DA at $37^{\circ} \mathrm{C}$ for $30 \mathrm{~min}$ in the dark. The DCFH-DA-fluorescence intensities (representing the intracellular ROS levels) were determined using a High Content Screening System (PerkinElmer, Massachusetts, USA). The average fluorescence intensities of DCFH-DA in the cells in 12 randomly chosen fields were analyzed using the Operetta ${ }^{\circledR}$ High Content Imaging System (PerkinElmer, Massachusetts, USA).

\section{Immunoprecipitation}

For immunoprecipitation experiments, total homogenates from H9c2 cells were treated with RIPA lysis buffer (P0013B, Beyotime Biotechnology, China), vortexed for $30 \mathrm{~s}$ and centrifuged for $10 \mathrm{~min}$ at $12000 \mathrm{r} / \mathrm{min}$. A total of $500 \mu \mathrm{g}$ of the cell extracts was subjected to immunoprecipitation with $1 \mu \mathrm{g}$ PGC-1a primary antibody at $4{ }^{\circ} \mathrm{C}$ overnight. The antibodybound proteins were precipitated with $15 \mu \mathrm{L}$ protein $\mathrm{G}$ magnetic beads (Cell Signaling Technology, \#8740) and rotated for $1-2 \mathrm{~h}$ at $37^{\circ} \mathrm{C}$. The beads were then gently centrifuged for 30 $\mathrm{s}$ at $1000 \mathrm{r} / \mathrm{min}$. After three RIPA buffer washes, the immunoprecipitates were diluted with $40 \mu \mathrm{L}$ of $5 \times$ sodium dodecyl sulfate (SDS) loading buffer and boiled at $95-100^{\circ} \mathrm{C}$ for $5 \mathrm{~min}$ to separate complexes from the protein $G$ magnetic beads ${ }^{[31]}$. The samples were then subjected to $10 \%$ SDS-polyacrylamide agarose gel electrophoresis (SDS-PAGE) and transferred to polyvinylidene difluoride (PVDF) membranes (Bio-Rad, USA). After blocking with 5\% skim milk, the membranes were incubated with an anti-acetylated-lysine antibody (Cell Signaling Technology, \#9441) overnight at $4^{\circ} \mathrm{C}$, washed in TBST three times and incubated with a secondary goat anti-rabbit polyclonal antibody at room temperature for $1 \mathrm{~h}$ (A0208, Beyotime, China). Finally, the signals were tested by an ECL kit (P0018A, Beyotime Biotechnology, China).

\section{RNA extraction and RT-PCR}

To extract total RNA, the heart tissues $(30 \mathrm{mg}$ ) or cultured cardiomyocytes were homogenized in $500 \mu \mathrm{L}$ TRIzol $^{\circledR}$ reagent (Invitrogen, USA) in a homogenizer. All procedures were completed according to previously reported protocols ${ }^{[32,33]}$. The total RNA pellet was dissolved in diethylpyrocarbonate (DEPC) water. The quality and quantity of total RNA were detected by ultraviolet absorption (optical density, $260 \mathrm{~nm} / 280$ $\mathrm{nm}$ ) using a NanoDrop 2000 (Thermo Scientific, USA). An equal amount of total RNA (500 ng) were reverse transcribed to cDNA using the PrimeScript ${ }^{\mathrm{TM}}$ 1st Strand cDNA Synthesis Kit (TaKaRa, Shiga, Japan). PCR amplification was performed using primers specific for rat RNA (Table 1) in a PCR amplifier (Applied Biosystems, Singapore). The cDNAs were amplified for 22-28 cycles. The reaction condition of amplification was performed at $95^{\circ} \mathrm{C}$ for $5 \mathrm{~s}, 56^{\circ} \mathrm{C}$ for $30 \mathrm{~s}$ and $72^{\circ} \mathrm{C}$ for $30 \mathrm{~s}$ (TaKaRa, Japan). The reaction products $(20 \mu \mathrm{L})$ were resolved on $1 \%$ agarose gels in $1 \times$ TAE buffer $(40 \mathrm{mmol} / \mathrm{L}$
Tris acetate and $1 \mathrm{mmol} / \mathrm{L}$ EDTA) and $6 \times$ loading buffer (Thermo scientific, USA). The reaction products were visualized using ethidium bromide staining and a Molecular Imager $^{\mathrm{R}} \mathrm{ChemiDoc}^{\mathrm{TM}}$ XRS+System (Bio-Rad Laboratories, Hercules, CA, USA). GAPDH served as an internal control.

\section{Western blotting analysis}

Heart tissue lysates were homogenized with RIPA buffer as described previously ${ }^{[34]}$. In brief, the cardiac tissue was homogenized in RIPA buffer (P0013B, Beyotime Biotechnology, China) containing the protease inhibitor phenylmethanesulfonyl fluoride (PMSF). Total protein concentrations were tested by a BCA protein assay kit (P0012, Beyotime Biotechnology, China). All samples were adjusted to the same volume with $5 \times$ SDS-PAGE sample buffer. Equal amounts of total proteins from rat hearts $(30 \mu \mathrm{g})$ were separated by $10 \%$ SDSPAGE at $100 \mathrm{~V}$ for $2 \mathrm{~h}$ and then electrophoretically transferred to PVDF membranes. The membranes were blocked using $1 \times$ PBS containing $5 \%$ skim milk for $1 \mathrm{~h}$ and then incubated with polyclonal antibody against PGC-1a, SIRT1, UCP2 and GAPDH overnight at $4{ }^{\circ} \mathrm{C}$. After washing in TBST $(1 \times$ TBS and $0.05 \%$ Tween-20), the membrane was incubated with secondary polyclonal antibodies for $1 \mathrm{~h}$ at room temperature. After the membrane was washed again in TBST, the immune complexes were detected using the ECL-Plus ${ }^{\mathrm{TM}}$ chemiluminescent detection reagent (P0018A, Beyotime Biotechnology, China) and visualized with a Molecular Imager ${ }^{\mathrm{R}} \mathrm{ChemiDoc}^{\mathrm{TM}} \mathrm{XRS}+$ System (Bio-Rad Laboratories, Hercules, CA, USA).

\section{Statistical analysis}

Data were analyzed using Student's $t$-test and multiple-comparison ANOVA with SigmaPlot 12.0 statistical software. All values are presented as the mean \pm standard error of the mean (SEM) from at least 3 separate experiments or determinations. A difference of $P<0.05$ was considered to be statistically significant.

\section{Results}

\section{Animal characterization}

In our study, a high-fat diet (HFD) combined with a single injection of STZ $(35 \mathrm{mg} / \mathrm{kg})$ in SD rats led to high plasma glucose levels ( $>16 \mathrm{mmol} / \mathrm{L})$ for $95 \%$ of the animals tested, while plasma glucose levels remained within the normal range in vehicle-injected control animals (Table 1). After 16 weeks, hyperglycemic rats developed type 2 diabetes characterized by polydypsia, polyurea, elevated $\mathrm{HbA1c}$ and dyslipidemia. The results showed that fasting plasma glucose (FBG) and $\mathrm{HbA1c}$ levels were both significantly increased in diabetic rats. However, RSV treatment inhibited rapid elevation of FBG and $\mathrm{HbA1c}$ levels, although these values were not within the normal ranges. Moreover, we also measured TG, TC, LDL and HDL levels in serum of different animals. The data in Table 2 show that HFD combined with a single injection of STZ substantially increased TG, TC and LDL levels and reduced HDL levels. RSV treatment significantly improved these parameters, although these values in the T2DM+RSV group were 
Table 1. PCR primer sequences.

\begin{tabular}{lll}
\hline Genes & Sense strand & Anti-sense strand \\
\hline PGC-1 $\alpha$ & $5^{\prime}$-CGCAACATGCTCAAGCCAAA-3' & $5^{\prime}$-GCGGTCTCTCAGTTCTGTCC-3' \\
NRF & $5^{\prime}$-ACGGCAGCTGAGGTAC-3' & $5^{\prime}$-TGAGTTCCGAAGCAACCGT-3' \\
UCP2 & $5^{\prime}$-TCTTCTGGGAGGTAGCAGGA-3' & $5^{\prime}$-GCTCTGAGCCCTTGGTGTAG-3' $^{\prime}$ \\
COX 1 & $5^{\prime}$-GCCTAGATGTAGACACCCGAGCC-3' & $5^{\prime}$-CGACGAGGTATCCCTGCTAATCC-3' \\
ANF & $5^{\prime}$-CTCCTTCTCCATCACCAAG-3' & $5^{\prime}$-AAGAAGGCAGATCTATCG-3' \\
BNP & $5^{\prime}$-GAGAGAGCAGGACACCATCG-3' & $5^{\prime}$-AAAACAACCTCAGCCCGTCA-3' \\
B-MHC & $5^{\prime}$-CCAACACCAACCTGTCCAA-3' & $5^{\prime}$-ACTCTTCATTCAGGCCCTTG-3' \\
GAPDH & $5^{\prime}$-AACTTTGGCATTGTGGAAGG-3' & $5^{\prime}$-TGTGAGGGAGATGCTCAGTG-3' \\
\hline
\end{tabular}

Table 2. Changes in fasting plasma glucose, glycohemoglobin and lipid profiles in diabetic rats. Data are presented as mean \pm SEM of at least 5 animals in each group. Animals were unanesthetized to obtain these variables. ${ }^{*} P<0.05,{ }^{* *} P<0.01$ vs control group. ${ }^{*} P<0.05$ vs T2DM group.

\begin{tabular}{|c|c|c|c|c|}
\hline Groups & Control & T2DM & T2DM+RSV & RSV \\
\hline $\mathrm{FBG}(\mathrm{mmol} / \mathrm{L})$ & $4.47 \pm 0.21$ & $26.07 \pm 1.85^{* *}$ & $16.89 \pm 3.58^{* * \#}$ & $4.31 \pm 0.62$ \\
\hline $\mathrm{HbA1c}$ & $10.16 \pm 0.50$ & $25.99 \pm 3.97^{* *}$ & $16.37 \pm 3.87^{* \#}$ & $9.68 \pm 1.20$ \\
\hline TG (mmol/L) & $0.45 \pm 0.09$ & $2.89 \pm 0.68^{* *}$ & $1.76 \pm 0.65^{* * \#}$ & $0.42 \pm 0.09$ \\
\hline $\mathrm{TC}(\mathrm{mmol} / \mathrm{L})$ & $1.39 \pm 0.20$ & $7.89 \pm 1.90^{* *}$ & $4.10 \pm 1.56^{\text {\#\# }}$ & $1.42 \pm 0.60$ \\
\hline LDL (mmol/L) & $0.23 \pm 0.04$ & $3.86 \pm 0.79^{* *}$ & $1.69 \pm 0.98^{* * \#}$ & $0.21 \pm 0.06$ \\
\hline
\end{tabular}

FBG: fasting blood glucose; TC: cholesterol; TG: triglyceride; HDL: high density lipoprotein; LDL: low density lipoprotein.

still higher than those in the control group. RSV administration had a small and insignificant effect on HDL levels. These results indicate that RSV has the potential to improve hyperglycemia and lipid disorders in type 2 diabetic rats.

\section{Resveratrol alleviated cardiac dysfunction and hypertrophy in diabetic hearts}

To determine whether cardiac function was improved by RSV treatment in long-term diabetes, echocardiography was performed in all groups at 16 weeks after STZ injection. After 16 weeks post-STZ injection, echocardiography revealed poor values for cardiac performance, including LVIDs, LVIDd, E/A, LVEF and LVFS (Figure 1A, 1C-1G). The cardiac functional parameters examined were lower in diabetic rats than in control rats as follows: E/A ratios $(0.76 \pm 0.11$ vs $1.40 \pm 0.03$, $P<0.01$, Figure 1E), LVFS (32.20 \pm 3.80 vs $42.11 \pm 5.00, P<0.01$, Figure $1 F)$ and LVEF (55.60 \pm 5.50 vs $70.30 \pm 9.10, P<0.01$, Figure $1 \mathrm{G})$. Compared with vehicle groups, RSV treatment for 16 weeks improved the attenuated $\mathrm{E} / \mathrm{A}$ ratios $(1.23 \pm 0.12$ vs $0.76 \pm 0.11, P<0.01$, Figure 1E), LVFS $(41.50 \pm 4.10$ vs $32.20 \pm 3.80$,

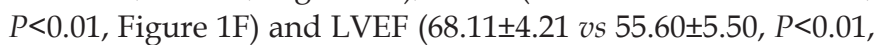
Figure $1 G$ ) in diabetic rats. To determine whether hyperglycemia and hyperlipidemia induced cardiac hypertrophy, LVIDs and LVIDd were investigated. LVIDs and LVIDd were both significantly increased in diabetic rats $(5.14 \pm 0.54$ vs $3.10 \pm 0.71$, $P<0.01$, Figure $1 \mathrm{C}$ or $7.70 \pm 0.41$ vs $5.52 \pm 0.87, P<0.01$, Figure 1D), whereas RSV treatment for 16 weeks restored structural abnormalities $(3.70 \pm 0.21$ vs $5.14 \pm 0.54, P<0.01$, Figure $1 C$ or
$6.20 \pm 0.50$ vs $7.70 \pm 0.41, P<0.01$, Figure 1D). Moreover, histological analysis by $H \& E$ staining of cardiac sections showed disordered cardiac muscle fibers and increased cross-sectional areas (CSAs) in cardiomyocytes from diabetic rats (Figure 1B), which was concomitant with significantly increased heart weight to body weight ratios ( $\mathrm{HW} / \mathrm{BW}, 3.85 \pm 0.56$ vs $2.32 \pm 0.23$, $P<0.01$, Figure $1 \mathrm{H})$, suggesting cardiac hypertrophy and remodeling. RSV treatment decreased heart weight to body weight ratios $(2.98 \pm 0.35$ vs $3.85 \pm 0.56, P<0.05$, Figure $1 \mathrm{H})$ and reversed cardiac hypertrophy induced by a high-fat diet and STZ injection. These histological changes were further confirmed by molecular marker analysis. The cardiac transcription levels of genes related to myocardial hypertrophy, including ANP, BNP and $\beta$-MHC, were substantially elevated in diabetic rats (Figure 1I, 1J). However, expression of all these genes was decreased upon RSV treatment compared with that in diabetic hearts. Taken together, these results indicate that RSV treatment blocked diabetes-induced cardiac dysfunction and hypertrophy.

\section{Resveratrol attenuates diabetes-induced oxidative stress in hearts}

The redox status was assessed by the endogenous antioxidant enzymes and lipid peroxidation products using biochemical measurements. SOD enzyme activity is decreased under oxidative stress conditions. As expected, we found dramatically reduced activities of antioxidant $S O D$ enzymes in diabetic hearts (Figure 2A) accompanied by significantly increased 
A

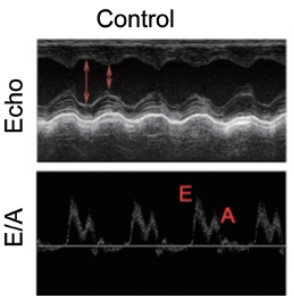

B
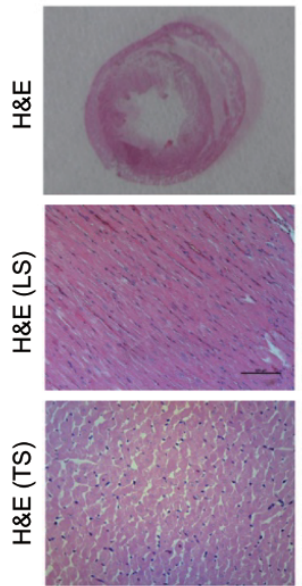

C

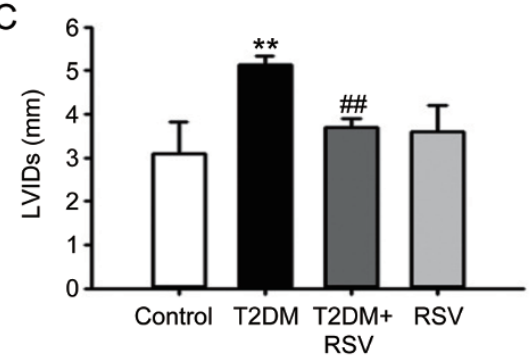

F

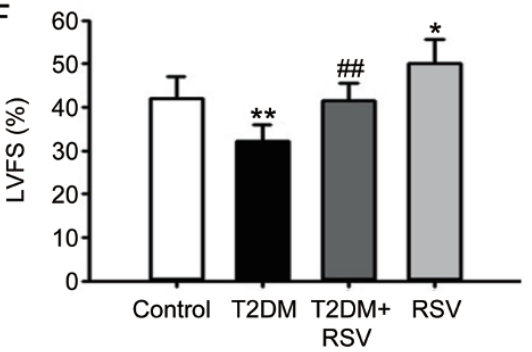

T2DM
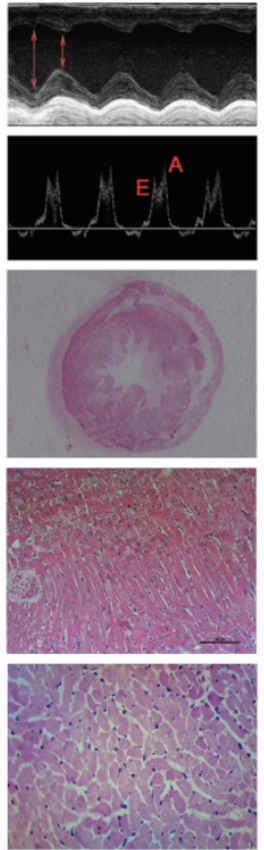

D
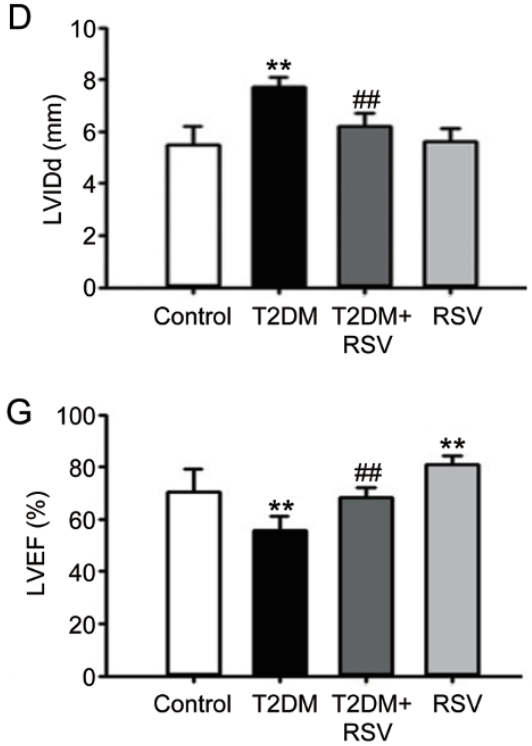

RSV
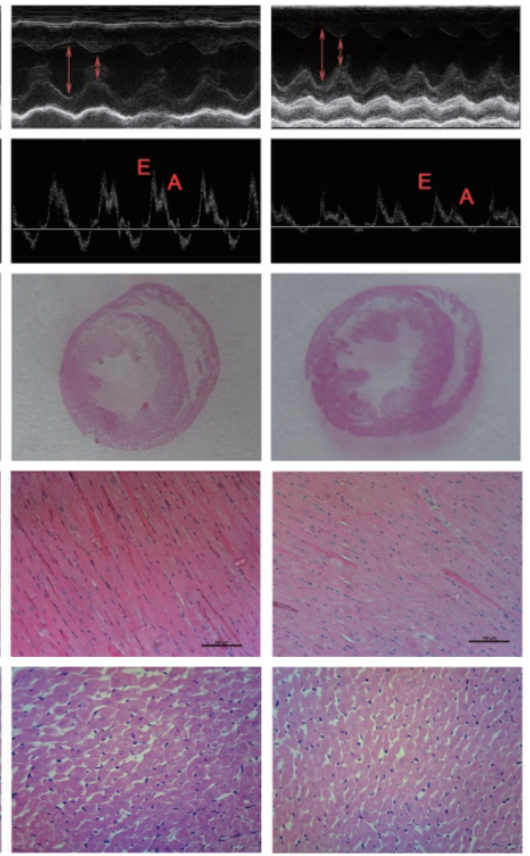
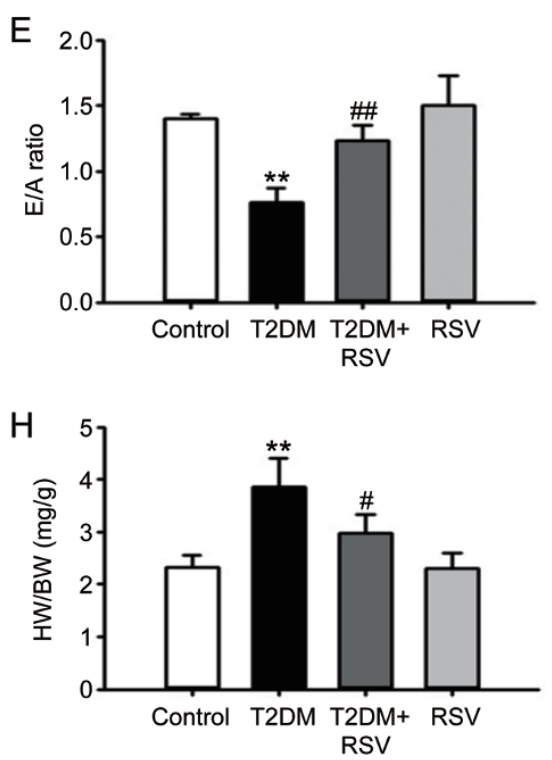

I
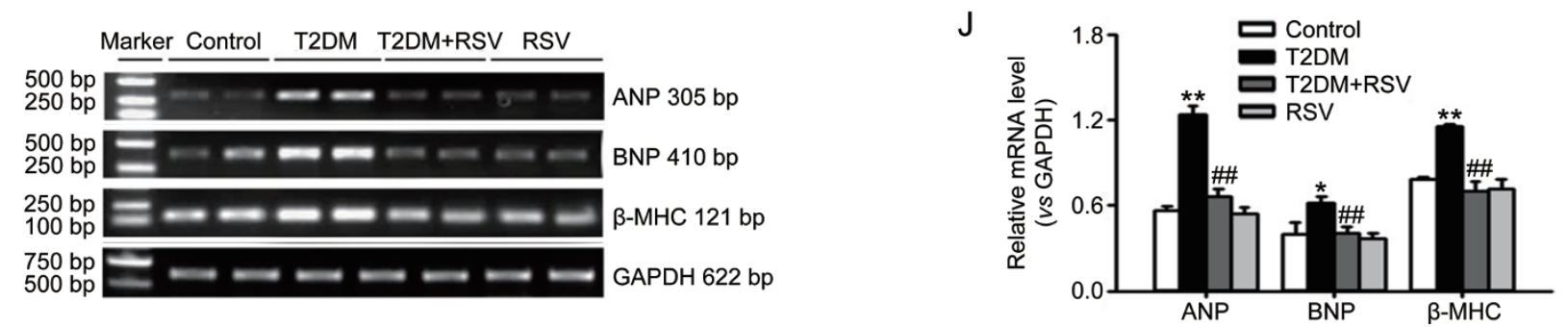

Figure 1. Resveratrol ameliorates cardiac dysfunction and hypertrophy in type 2 diabetic hearts. (A) Transthoracic echocardiography in rats at 16 weeks. (B) H\&E-stained longitudinal sections (LS), transverse sections (TS) of rat left ventricles; scale bar, $100 \mu \mathrm{m}$; (C-H) Assessments of LV internal dimension at systole (LVIDs) (C), left ventricular internal dimension at diastole (LVIDd) (D), ratio of peak E to peak $A(E / A)$ (E), Left ventricular fraction shortening (LVFS\%) (F), left ventricular ejection fraction (LVEF\%) (G) and heart weight to body weight (HW/BW) (H). (I, J) Heart tissues from rats were individually processed for mRNA extraction and RT-PCR, GAPDH was used as loading control. The mRNA transcriptions of ANP, BNP and $\beta$-MHC were determined ( $n=4$ per group). Data are presented as mean \pm SEM. ${ }^{*} P<0.05,{ }^{* *} P<0.01$ vs control group. ${ }^{\#} P<0.05,{ }^{\# \#} P<0.01$ vs T2DM group. 
A

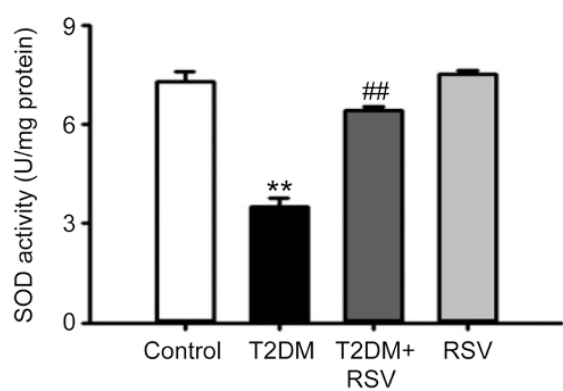

C
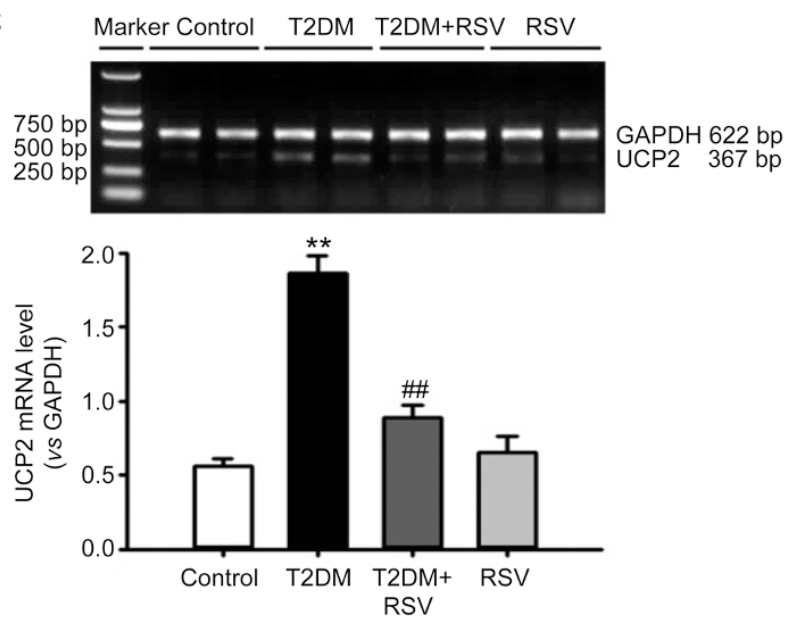

B

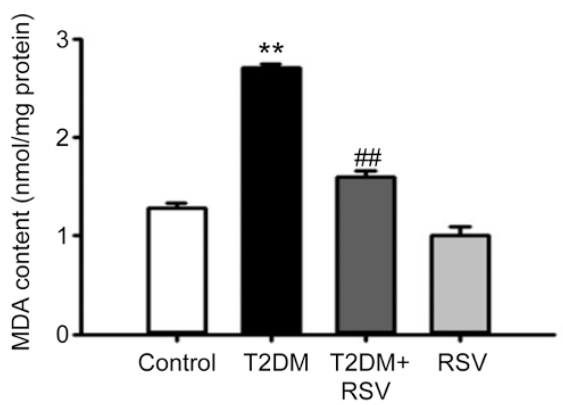

D
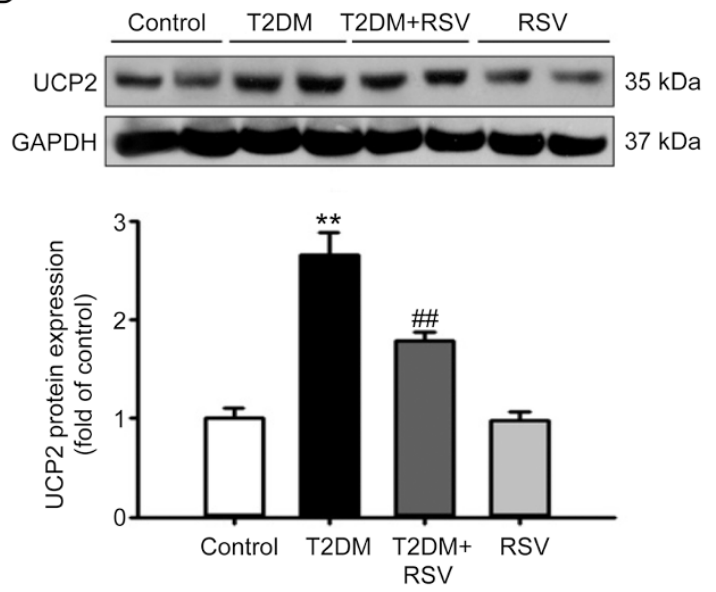

Figure 2. Resveratrol ameliorates oxidative stress in diabetic hearts. Biochemical measurement was performed guided by manipulation instructions to detect the myocardial levels of SOD activity (A) and lipid peroxidation product (MDA) (B), RSV rescued the reduced SOD activity and increased MDA levels in diabetic hearts. (C) Total mRNA was extracted from heart tissues of rats and RT-PCR was performed to examine UCP2 transcriptions ( $n=4$ per group). GAPDH was used as loading control. RSV therapy prevented the increased UCP2 mRNA levels in diabetes. (D) Western blotting was used to detect UCP2 protein expressions. Representative radiograms obtained from all groups of animals. GAPDH was used as a loading control. ImageJ software were used to measure band intensity and each band intensity was normalized to GAPDH. Data are expressed as mean \pm SEM of $4-5$ rats.

${ }^{* *} P<0.01$ vs control group. ${ }^{\# \#} P<0.01$ vs T2DM group.

MDA formation compared with the control group (Figure 2B). However, RSV treatment for 16 weeks abolished these alterations (Figure 2A, 2B).

We also measured uncoupling protein 2 (UCP2) expression, which is primarily expressed in inner mitochondrial membrane to regulate fatty acid oxidation, mitochondrial biogenesis and reactive oxygen species $(\mathrm{ROS})^{[35]}$. A previous study reported that down-regulation of UCP2 is associated with increased oxidative stress and impaired cardiovascular function $^{[36]}$. However, in our study, significantly elevated levels of UCP2 mRNA transcription and protein expression were observed in type 2 diabetic rats, indicating a feedback mechanism involving $\mathrm{UCP} 2$ regulation of cardiac oxidative stress or increased ROS production. RSV treatment diminished these effects (Figure 2C, 2D). These results suggest that RSV has an anti-oxidant effect on diabetes-induced oxidative stress.

Resveratrol improved mitochondrial function and biogenesis in diabetic hearts

Cardiac mitochondrial dysfunction contributes to diabetic cardiomyopathy, as shown by impaired ATP synthesis and mitochondrial biogenesis. We examined the ATP levels and mitochondrial copy number as determined by the ratio of cytochrome $c$ oxidase subunit I gene to $\beta$-actin (COX 1/ $\beta$-actin) to estimate mitochondrial function and biogenesis in heart tissues. The data in Figure 3 show a substantial reduction in ATP levels and COX $1 / \beta$-actin ratios of diabetic rats compared with those in age-matched controls, although RSV administration for 16 weeks reversed diabetes-induced cardiac mitochondrial dysfunction. These data were also confirmed by substantially decreased protein and transcript levels of several genes, such as PGC-1a and NRF, which were reported to regulate mitochondrial biogenesis and function. The RT-PCR results showed that the transcription levels of PGC-1a and NRF were sharply reduced in diabetic rats. However, RSV treatment significantly enhanced PGC-1a and NRF transcription. At the protein level, SIRT1 and PGC-1a expression were both downregulated, whereas these alterations were restored by RSV treatment for 16 weeks. These data strongly indicate that resveratrol has beneficial effects on diabetes-induced cardiac mitochondrial dysfunction. 
A

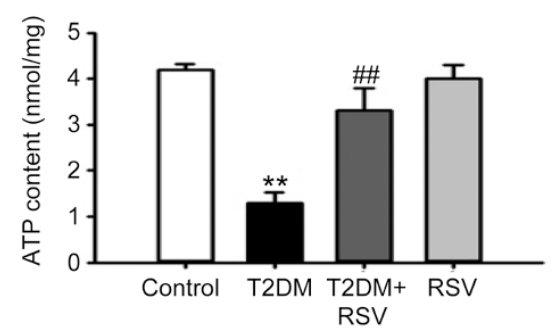

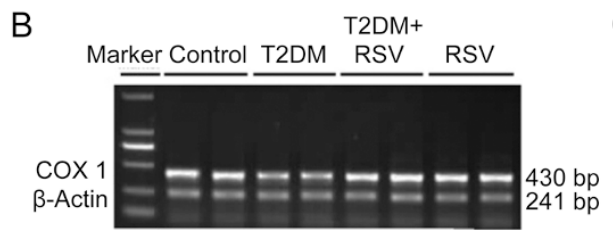
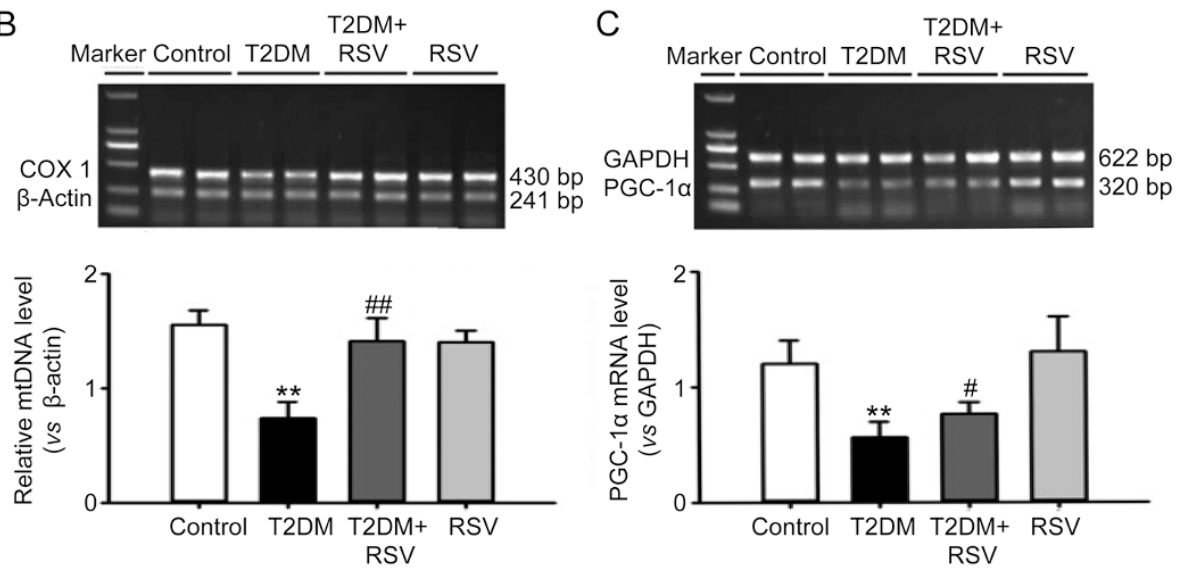

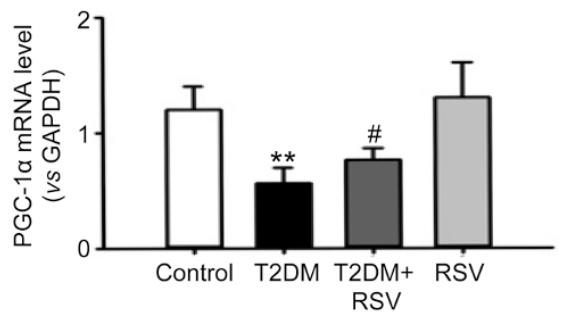

D
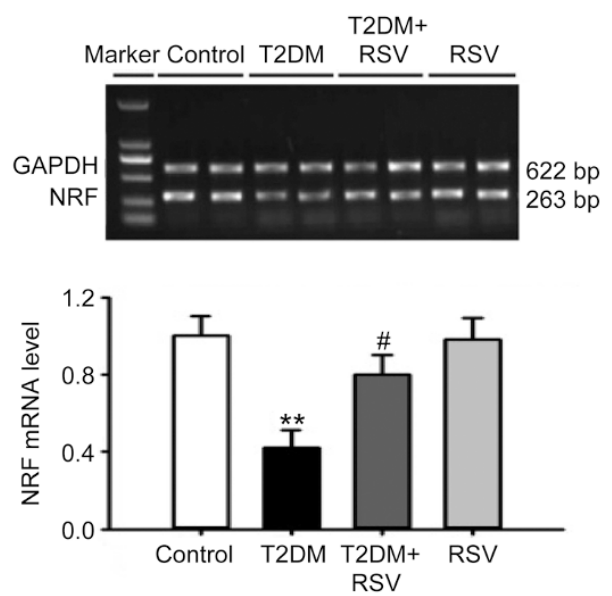

$\mathrm{E}$
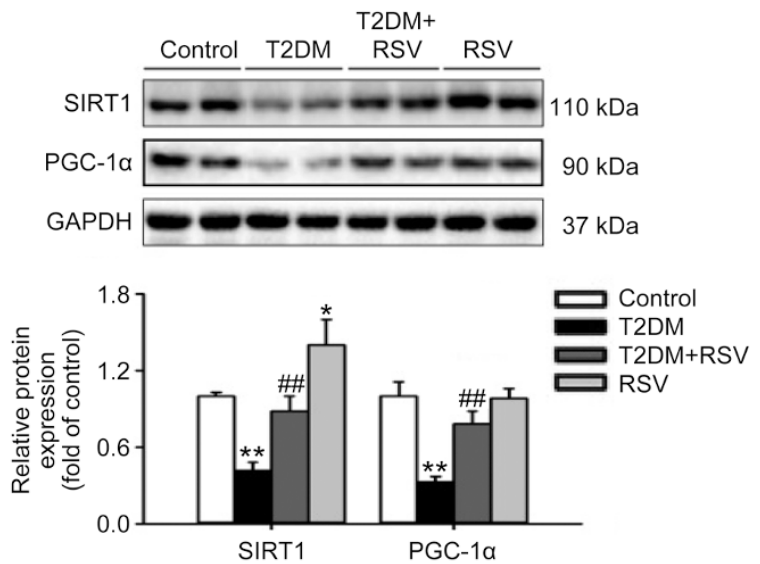

Figure 3. Resveratrol improved mitochondrial function and biogenesis in diabetic hearts. (A) Cardiac ATP levels were measured by ATP Assay Kit purchased from Beyotime after diabetic rat's model established and RSV treatment for 16 week. (B) Heart tissues from rats were individually processed for DNA extraction and then PCR, $\beta$-actin was used as loading control. The mtDNA levels of COX 1 was determined ( $n=4$ per group). (C, D) Heart tissues from rats were individually processed for mRNA extraction and RT-PCR, GAPDH was used as loading control. The mRNA levels of PGC-1 $\alpha$ (C) and NRF (D) were determined ( $n=4$ per group). (E) Western blotting; representative radiograms obtained from all groups of animals. GAPDH was used as a loading control. ImageJ software were used to measure band intensity and each band intensity was normalized to GAPDH. Data are expressed as mean \pm SEM of $4-5$ rats. ${ }^{* *} P<0.01$ vs control group. ${ }^{\#} P<0.05,{ }^{\# \#} P<0.01$ vs T2DM group.

High glucose induces insulin resistance in $\mathrm{H} 9 \mathrm{c} 2$ cells

To determine whether high-glucose-incubated cardiomyocytes show insulin resistance and are therefore representative for T2DM as in an in vivo setting, we detected expression of Akt phosphorylation at Serine 473 in cardiomyocytes incubated with $30 \mathrm{mmol} / \mathrm{L}$ glucose at different time points $(0,24$, 48,72 , and $96 \mathrm{~h}$ ), and $10 \mathrm{nmol} / \mathrm{L}$ insulin was added $10 \mathrm{~min}$ before cells were harvested to reflect cardiac insulin signaling pathways (Figure 4). The data showed that high glucose (30 mmol/L) suppressed phosphorylation of Akt S473 in cardiomyocytes in a time-dependent manner, suggesting impaired cardiac insulin signal transduction in high glucose-cultured cardiomyocytes, which is consistent with the in vivo setting.

SIRT1 is essential for resveratrol amelioration of high-glucoseinduced oxidative stress in $\mathrm{H} 9 \mathrm{c} 2$ cells

SIRT1 is ubiquitously expressed in multiple cell types in the cardiovascular system, including cardiomyocytes. To further evaluate whether the cardioprotection of RSV in the in vivo model is due to a direct effect on cardiomyocytes through SIRT1, we pretreated H9c2 cells with RSV (20 $\mu \mathrm{mol} / \mathrm{L})$ for $2 \mathrm{~h}$ and then incubated them with high concentrations of glucose (HG, $30 \mathrm{mmol} / \mathrm{L}$ ) or sirtinol (a SIRT1 inhibitor, $25 \mu \mathrm{mol} / \mathrm{L}$ ) for $48 \mathrm{~h}$. HG stimulation for $48 \mathrm{~h}$ reduced SOD activity by approximately threefold (Figure 5A) and increased MDA content by approximately fourfold (Figure 5B) in H9c2 cells, as well as sharply elevated ROS levels (Figure 5D, 5E). However, RSV treatment reversed these changes. Moreover, sirtinol, a SIRT1 inhibitor, abolished RSV-mediated protection in $\mathrm{H} 9 \mathrm{c} 2$ cells. In addition, the inhibition of HG-induced oxidative stress by RSV pretreatment was further confirmed by downregulation of UCP2 protein expression via Western blot assay (Figure 5C); sirtinol also abrogated the effects of RSV on UCP2 expression. These data suggest that RSV treatment directly 

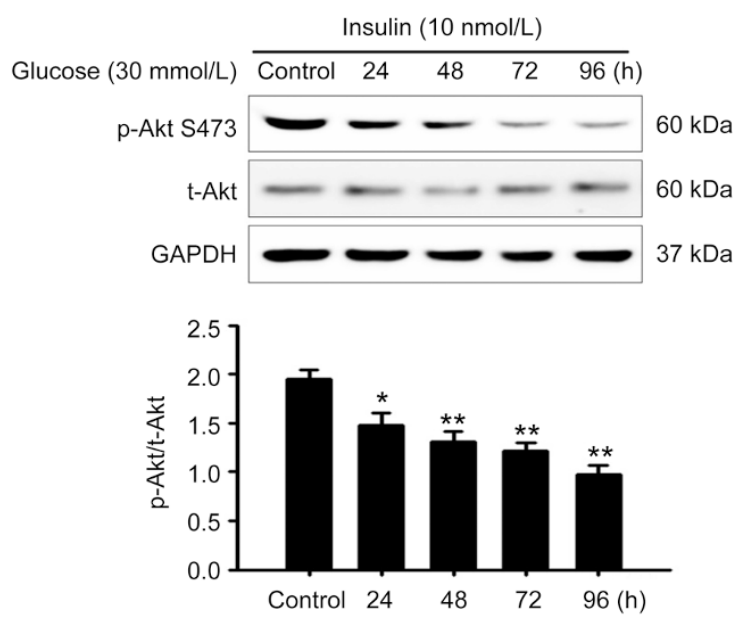

Figure 4. High glucose induces insulin resistance in $\mathrm{H9c2}$ cells. $\mathrm{H9c2}$ cells were incubated with $30 \mathrm{mmol} / \mathrm{L}$ glucose in different time courses $(0$, $24,48,72$, and $96 \mathrm{~h}$ ), and then $10 \mathrm{nmol} / \mathrm{L}$ insulin were added before 10 min cells harvested. Western blotting was performed to detect p-Akt (Ser 473) and total Akt expressions. GAPDH was used as a loading control. High glucose $(30 \mathrm{mmol} / \mathrm{L})$ suppressed phosphorylation of Akt S473 in a time-dependent manner, suggesting cardiac insulin resistance in high glucose cultured $\mathrm{H} 9 \mathrm{c} 2$ cells. Data are expressed as mean $\pm \mathrm{SEM}$ of three independent measurements. ${ }^{*} P<0.05,{ }^{* *} P<0.01$ vs control group. acts on cardiomyocytes to exert the antioxidant effect in a SIRT1-dependent manner.

SIRT1 is essential for resveratrol amelioration of high-glucoseinduced mitochondrial dysfunction in $\mathrm{H} 9 \mathrm{c} 2$ cells

We also tested the potential role of SIRT1 in resveratrolmodulated mitochondrial function and biogenesis in cultured $\mathrm{H} 9 \mathrm{c} 2$ cells treated with high glucose. RSV augmented myocardial ATP levels and mtDNA content (COX 1) (Figure 6A, $6 \mathrm{~B})$, whereas in the presence of sirtinol, these responses were partially blocked. Moreover, RSV also induced the expression of mitochondrial biogenesis factors, such as PGC-1a and NRF in vitro (Figure 6C, 6D), which were inhibited following treatment with sirtinol. In addition, high-glucose-treated H9c2 cells exhibited depolarized mitochondrial membrane potential $(\Delta \psi \mathrm{m})$, which was shown by a significant decrease in the JC-1 aggregate (red fluorescence)/JC-1 monomer (green fluorescence) ratio (Figure 6E, 6F). Resveratrol protected against mitochondrial membrane potential depletion caused by highglucose treatment, and this effect was also antagonized by the SIRT1 inhibitor sirtinol.

To further evaluate the requirement of SIRT1 in mitochondrial biogenesis in response to RSV, H9c2 cells were trans-
A
B

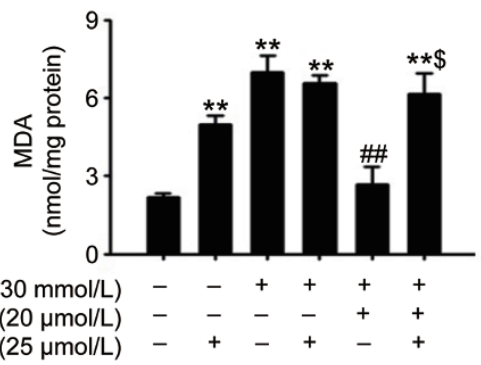

C

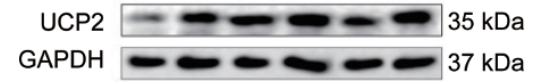

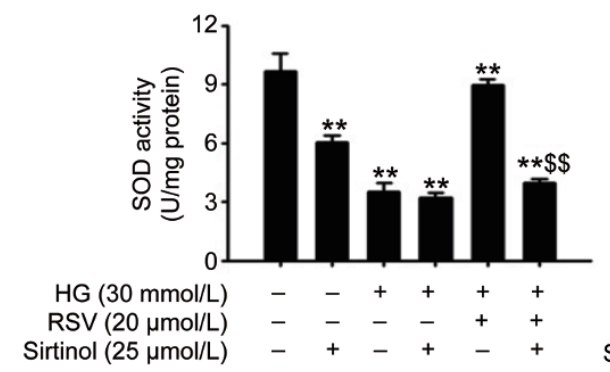

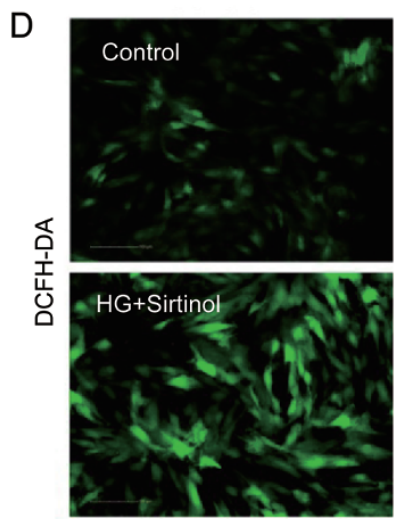

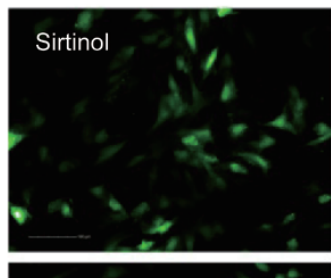

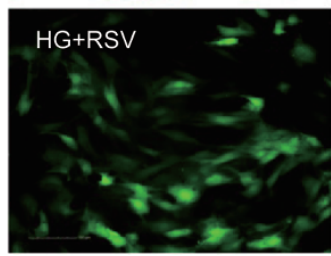

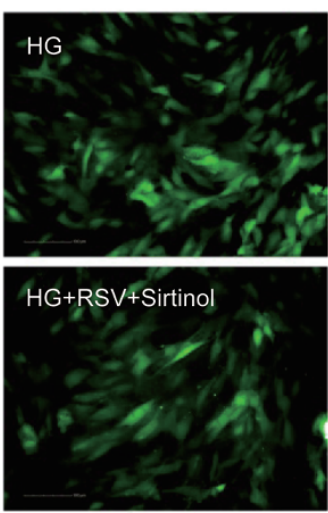

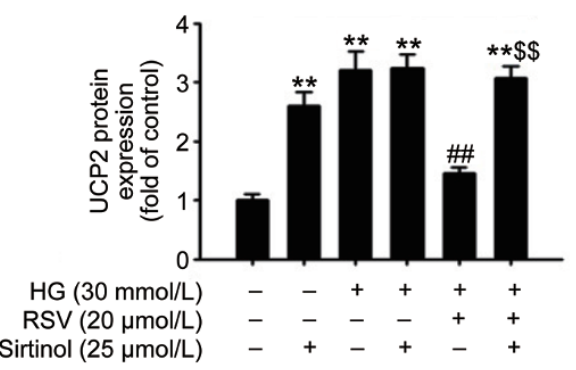

$E$

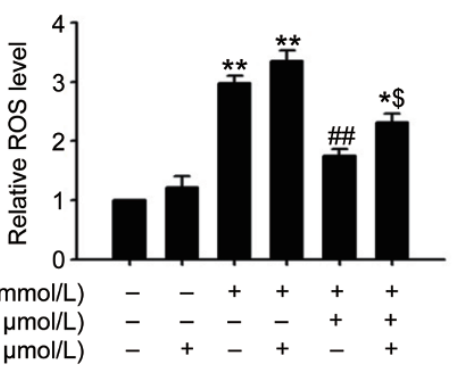

Figure 5. SIRT1 is essential for RSV ameliorates HG-induced oxidative stress in H9c2 cells. (A, B) Biochemical analysis of anti-oxidant enzyme activity (SOD) and lipid peroxidation products (MDA) levels in H9c2 cells with or without glucose (30 mmol/L), RSV (20 $\mu \mathrm{mol} / \mathrm{L})$ and Sirtinol (25 $\mu \mathrm{mol} / \mathrm{L})$ for $48 \mathrm{~h}$. (C) UCP2 and GAPDH protein levels were detected by Western blotting in H9c2 cells. (D) Intracellular ROS levels were detected in H9c2 cells by staining with DCFH-DA after treated with high glucose $(30 \mathrm{mmol} / \mathrm{L})$, RSV $(20 \mu \mathrm{mol} / \mathrm{L})$ and Sirtinol $(25 \mu \mathrm{mol} / \mathrm{L})$ for $48 \mathrm{~h}$; Scale bar, $100 \mu \mathrm{m}$. (E) The results of the statistical analysis of the relative intracellular ROS levels (represented by the mean intensity of DCFH-DA fluorescence). Data are expressed as mean \pm SEM of three independent measurements. ${ }^{*} P<0.05,{ }^{* *} P<0.01$ vs non-treated H9c2 cells. ${ }^{\# \#} P<0.01$ vs HG treated H9c2 cells, ${ }^{\$} P<0.05,{ }^{\$ \$} P<0.01$ vs $\mathrm{HG}+\mathrm{RSV}$ treated with $\mathrm{H} 9 \mathrm{c} 2$ cells. 

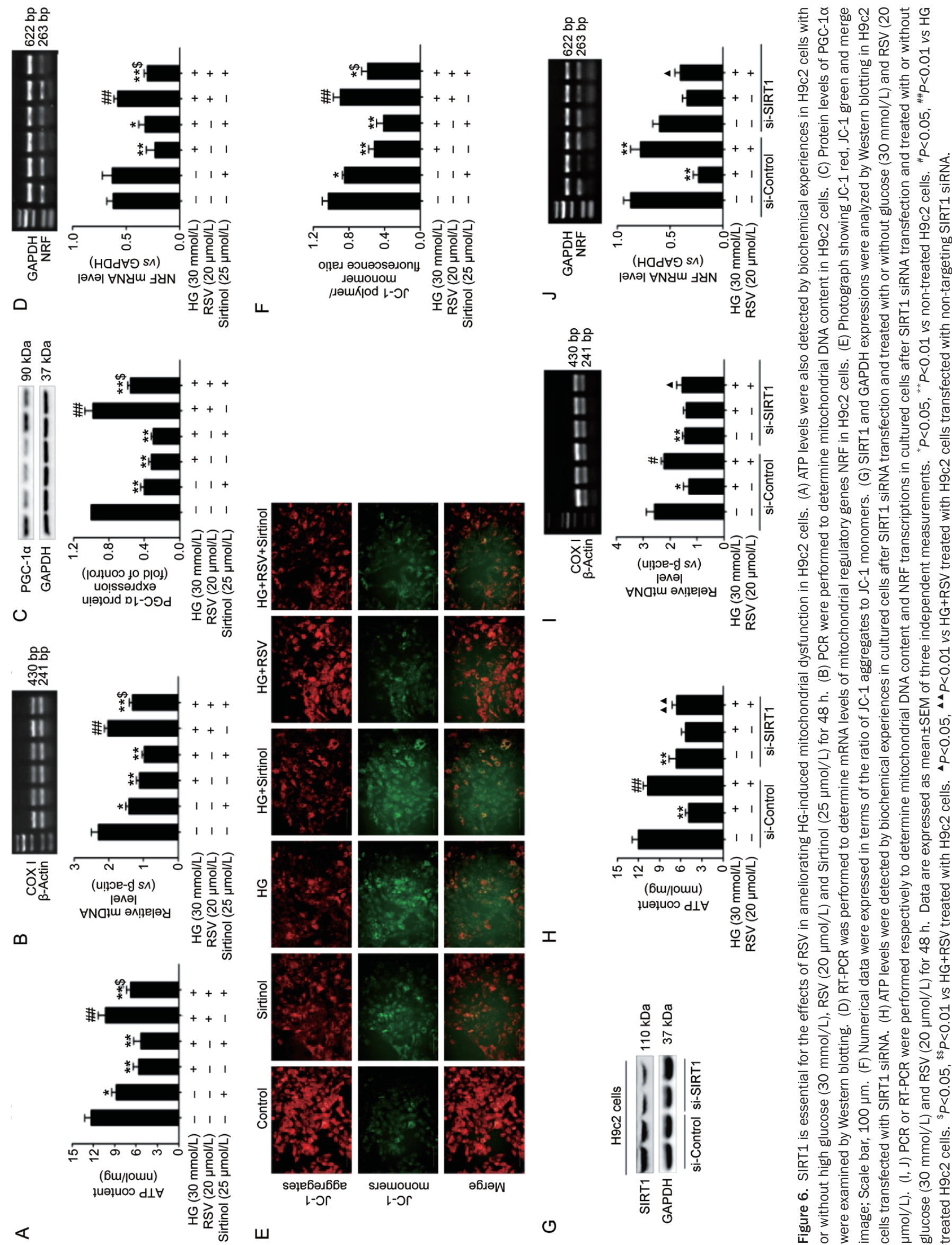
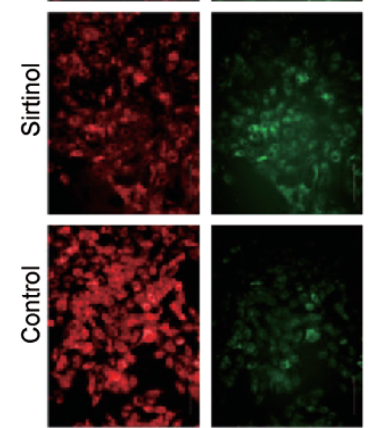

sәребอл66е

l-כr

ш
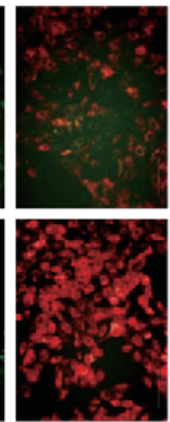

ә6อəพ

0

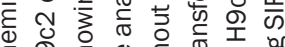

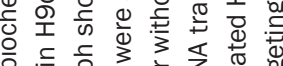

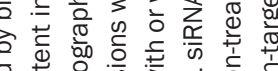

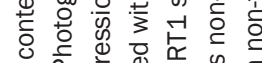

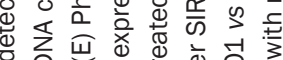

口

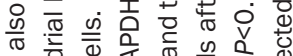

ข

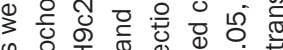

ब)

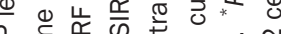

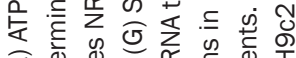

उ肯

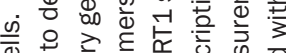

ن

오 훈

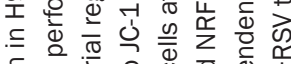

ᄃ

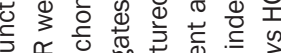

零赛员

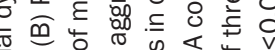

난

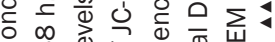

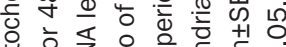

है

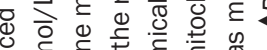

을 है है है है है

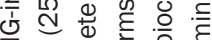

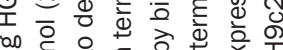

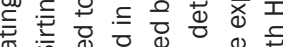

\% ज

ब

है

$\subseteq$ वे

के हो 3 का

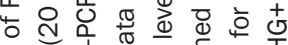

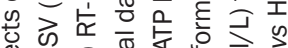

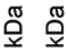

읃 응

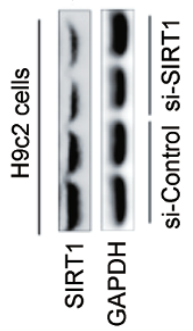

(1)

๗

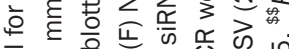

을 일

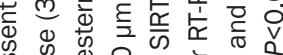

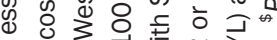

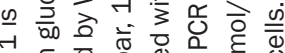

단

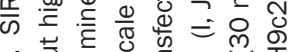

0 苛

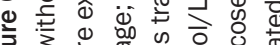

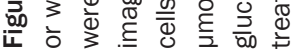




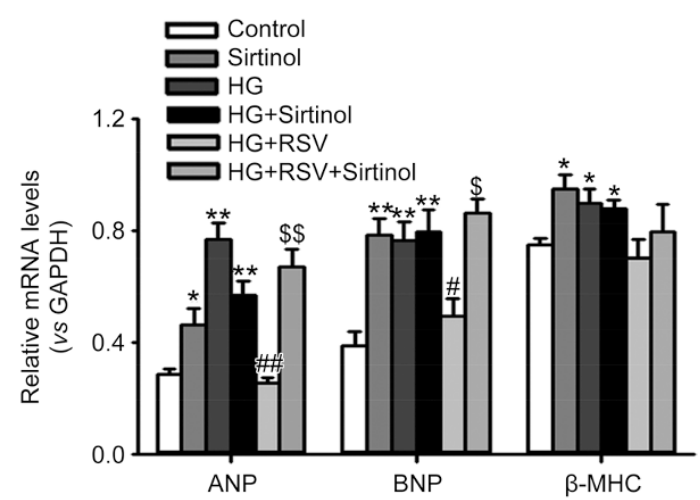

B
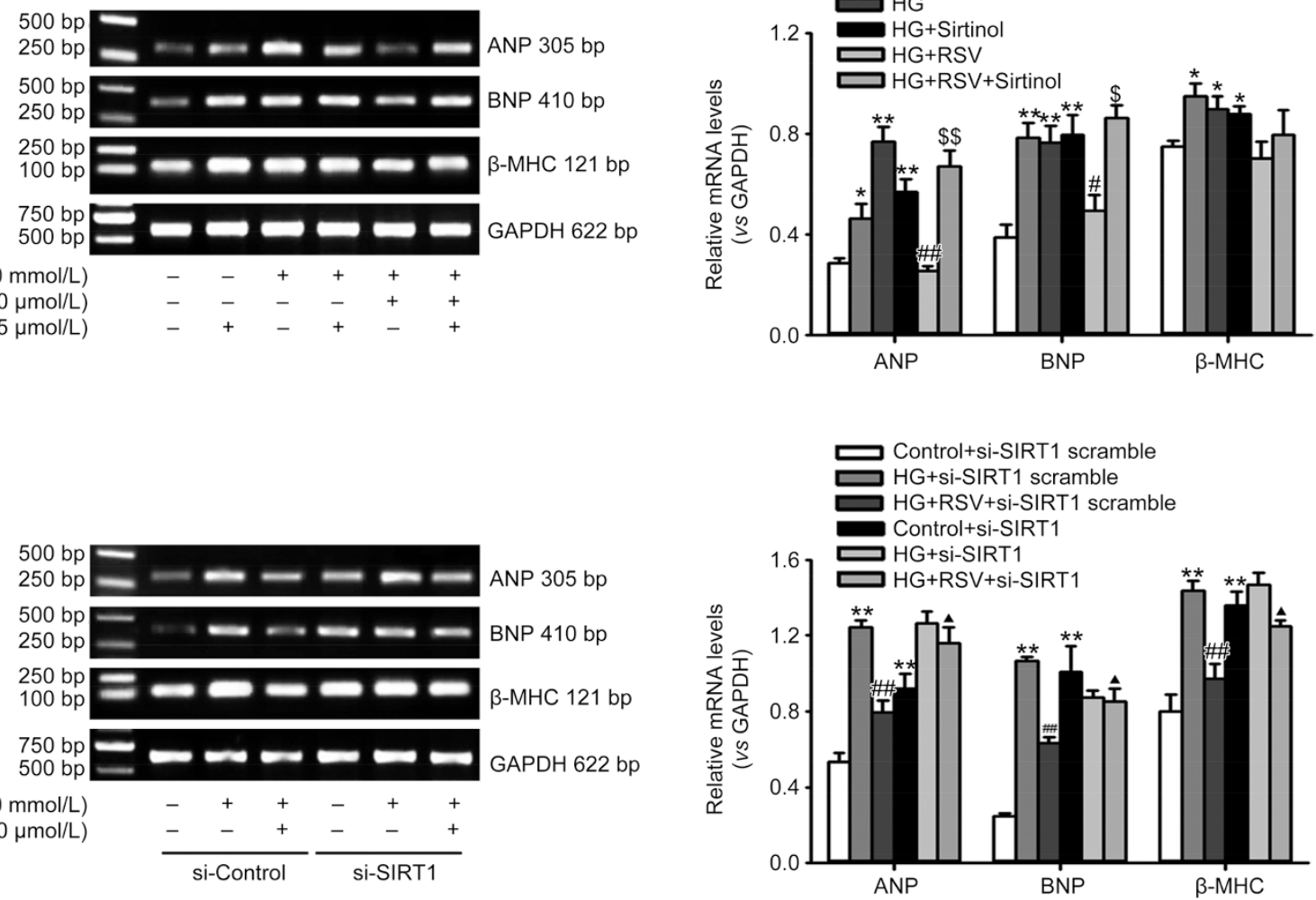

Figure 7. Resveratrol ameliorates hypertrophy in H9c2 cells depending on SIRT1. (A) Total mRNA was extracted from H9c2 cells, which were separately incubated with or without glucose $(30 \mathrm{mmol} / \mathrm{L})$, RSV $(20 \mu \mathrm{mol} / \mathrm{L})$ and Sirtinol $(25 \mu \mathrm{mol} / \mathrm{L})$ for $48 \mathrm{~h}$. RT-PCR was performed to examine pro-hypertrophy markers ANP, BNP and $\beta$-MHC transcriptions. (B) RT-PCR were performed in H9c2 cells after SIRT1 siRNA transfection and treated with or without glucose $(30 \mathrm{mmol} / \mathrm{L})$ and RSV $(20 \mu \mathrm{mol} / \mathrm{L})$ for $48 \mathrm{~h}$. GAPDH was used as loading control. Data are expressed as mean \pm SEM of three independent measurements. ${ }^{*} P<0.05,{ }^{* *} P<0.01$ vs non-treated H9c2 cells. ${ }^{\#} P<0.05,{ }^{\# \#} P<0.01$ vs HG treated H9c2 cells. ${ }^{\$} P<0.05,{ }^{\$ \$} P<0.01$ vs HG+RSV treated with H9c2 cells. ${ }^{\star} P<0.05$ vs HG+RSV treated with H9c2 cells transfected with non-targeting SIRT1 siRNA.

fected with SIRT1-specific siRNA and control (scramble) siRNA. The effectiveness of SIRT1 knockdown on SIRT1 protein levels was confirmed by Western blotting analysis (Figure 6G). Suppression of SIRT1 by siRNA knockdown abolished RSV-mediated $(20 \mu \mathrm{mol} / \mathrm{L})$ induction of mitochondrial biogenesis. In SIRT1 knockdown cells, there was a significant reduction in ATP and mtDNA content (Figure 6H, 6I), as well as decreased NRF mRNA expression (Figure 6J) elicited by RSV. These results demonstrate the involvement of SIRT1 during high-glucose-induced cardiomyocyte mitochondrial dysfunction and in mediating the RSV protective effects in mitochondria during diabetic cardiomyopathy.

Resveratrol ameliorates hypertrophy in H9c2 cells through SIRT1 The protective role of RSV in cardiac remodeling in vivo was supported by the observations of improved morphologic abnormalities and suppressed pro-hypertrophy markers. To determine whether RSV modifies hypertrophy in vitro under high-glucose conditions, ANP, BNP and $\beta$-MHC, markers of cardiac hypertrophy, were detected in H9c2 cells by RTPCR. The results showed that incubation with high glucose augmented transcription levels of ANP, BNP and $\beta$-MHC, whereas in the presence of RSV, these responses were ameliorated. However, this beneficial effect was blocked by addition of sirtinol, indicating that SIRT1 mediated RSV's antihypertrophic effects (Figure 7A). To further confirm these results, we used small interfering RNA-mediated knockdown of SIRT1 in H9c2 cells to examine the effect of reduced SIRT1 on RSV treatment (Figure 7B). A similar effect to that of sirtinol was observed, as SIRT1 knockdown diminished the RSVmediated reduction in transcription levels of ANP, BNP and $\beta$-MHC. Collectively, these data suggest that RSV is capable of blocking the high-glucose-induced hypertrophy in H9c2 cells in a SIRT1-dependent manner.

RSV treatment induces PGC-1 $\alpha$ deacetylation in a SIRT1dependent manner in $\mathrm{H} 9 \mathrm{c} 2$ cells

We next investigated the potential mechanisms of RSV in protecting mitochondrial function during hyperglycemia. We performed in vitro experiments using cultured H9c2 cells. After $2 \mathrm{~d}$ of culture in normal medium, we exposed H9c2 cells with high-glucose conditions $(30 \mathrm{mmol} / \mathrm{L})$ with or without RSV $(20 \mu \mathrm{mol} / \mathrm{L})$ and sirtinol $(25 \mu \mathrm{mol} / \mathrm{L})$ and found that H9c2 cells showed a significant HG-induced downregulation 
A

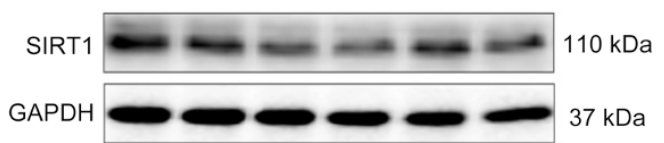

B

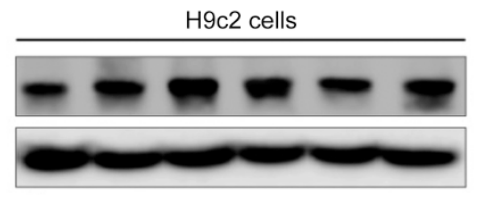

P: PGC-1a

WB: Ac-lysine

IP: PGC-1a WB: PGC-1a

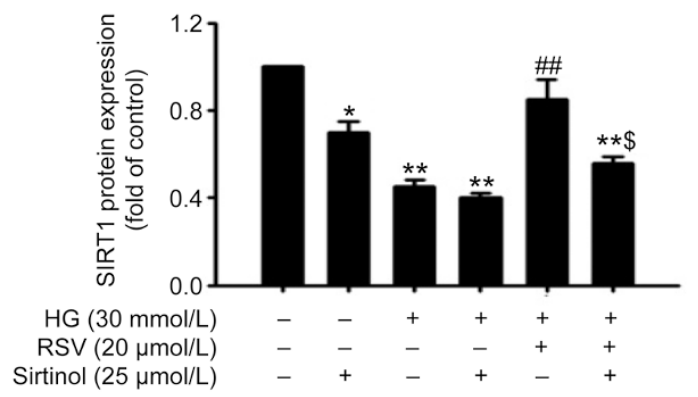

C

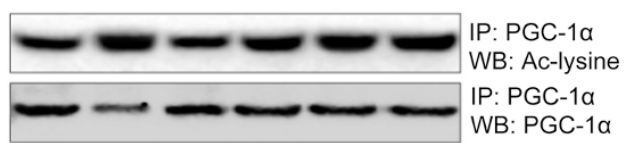

HG (30 mmol/L)

$\operatorname{RSV}(20 \mu \mathrm{mol} / \mathrm{L})$

Sirtinol $(25 \mu \mathrm{mol} / \mathrm{L})$
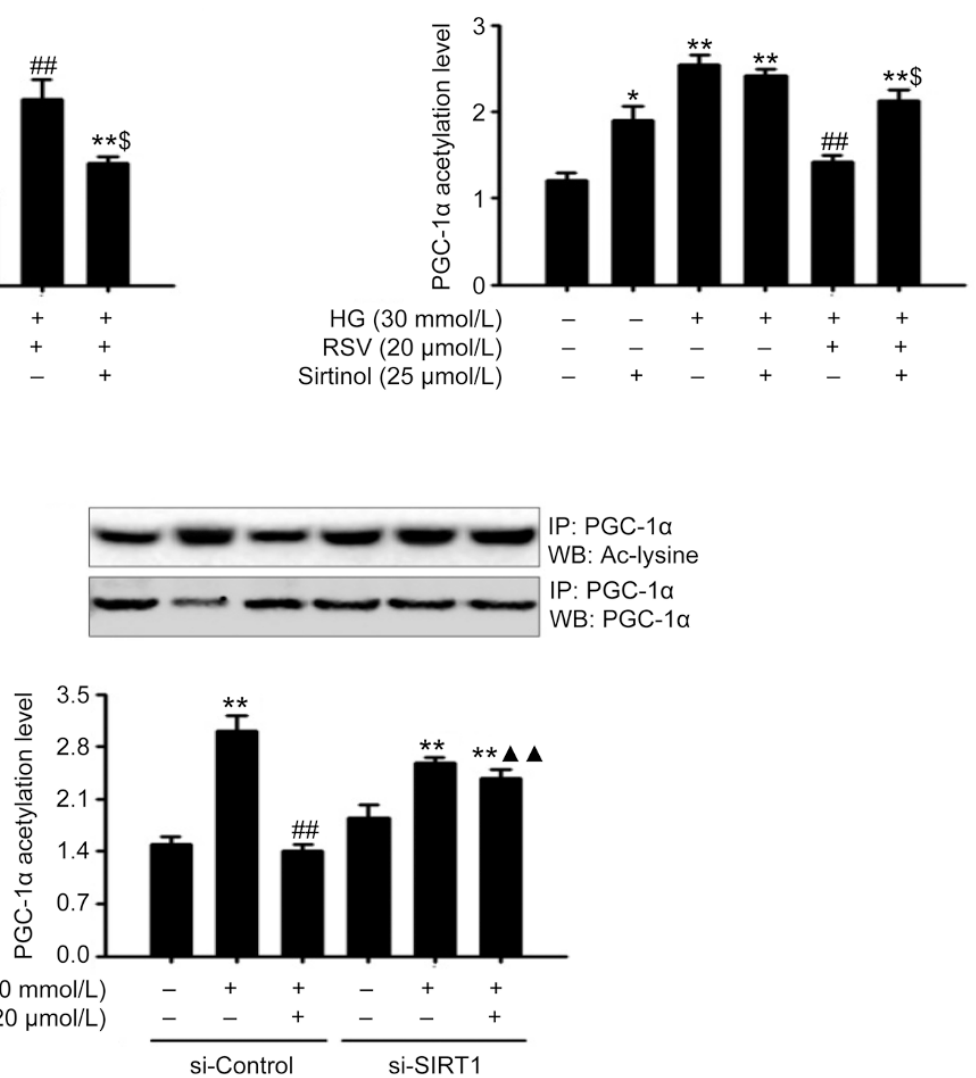

Figure 8. RSV induces PGC-1 $\alpha$ deacetylation in a SIRT1-dependent manner in H9c2 cells. (A) Western blotting to detect SIRT1 expression in H9c2 cells and quantification of band density showing significant reduction of SIRT1 expression after treatment with $\mathrm{HG}(30 \mathrm{mmol} / \mathrm{L})$ and sirtinol $(25 \mu \mathrm{mol} / \mathrm{L})$, while RSV $(20 \mu \mathrm{mol} / \mathrm{L})$ augmented SIRT1 expression. (B) Acetylation level of PGC-1 $\alpha$ was evaluated by performing immunoprecipitation in H9c2 cells after treatment with HG (30 mmol/L), RSV $(20 \mu \mathrm{mol} / \mathrm{L})$ or sirtinol $(25 \mu \mathrm{mol} / \mathrm{L})$, and quantification of band density supported that HG induced hyperacetylation of PGC-1 $\alpha$ and RSV reduced PGC-1 $\alpha$ acetylation level depending on SIRT1. (C) Acetylation level of PGC-1 $\alpha$ was evaluated by performing immunoprecipitation after SIRT1 siRNA transfection in $\mathrm{H} 9 \mathrm{c} 2$ cells with or without $\mathrm{HG}(30 \mathrm{mmol} / \mathrm{L})$ and resveratrol $(20 \mu \mathrm{mol} / \mathrm{L})$ for $48 \mathrm{~h}$. Data are presented as mean \pm SEM of three independent measurements. ${ }^{*} P<0.05,{ }^{* *} P<0.01$ vs non-treated H9c2 cells. ${ }^{\#} P<0.05,{ }^{\#} P<0.01$ vs HG treated H9c2 cells. ${ }^{\$} P<0.05$ vs HG+RSV. ${ }^{\Delta \mathbf{\Delta}} P<0.01$ vs HG+RSV cells transfected with non-targeting sirt1 siRNA.

of SIRT1 protein expression. Resveratrol augmented the SIRT1 expression in high-glucose conditions, whereas in the presence of sirtinol, this response was partially blocked (Figure 8A). SIRT1 is a multifaceted NAD+-dependent protein deacetylase that acts on a variety of proteins and regulates mitochondrial function, stress resistance and genomic integrity. PGC-1a has been reported as a target of SIRT1. Therefore, we hypothesized that the protective effects of RSV on cardiac mitochondrial function under high-glucose conditions may be associated with SIRT1-regulated PGC-1a deacetylation. To test this hypothesis, immunoprecipitation was performed to determine the acetylation levels of PGC-1a, and the results showed that PGC-1a acetylation was significantly higher in the highglucose medium than in the normal medium. In contrast, RSV protected against the increased HG-induced hyperacetylation of PGC-1a, and this effect was blocked by sirtinol (Figure 8B). To confirm that these effects were due to SIRT1 inhibition, we knocked down SIRT1 expression using siRNA in cultured H9c2 cells. Similar to the results observed following addition of sirtinol, SIRT1 knockdown abrogated the deacetylation effects of RSV on PGC-1a in HG-treated H9c2 cells. Furthermore, we also unexpectedly found that SIRT1 knockdown enhanced PGC-1a acetylation levels in H9c2 cells under normal conditions, indicating that SIRT1 participates in regulating PGC-1a activity not only when cells are exposed to pathologic conditions but also under physiological conditions (Figure $8 \mathrm{C}$ ). Taken together, these data suggested that signaling via SIRT1 and PGC-1a deacetylation may be responsible for the 
protective effects of RSV during diabetic cardiomyopathy.

\section{Discussion}

Overall, our in vivo and in vitro experiments recapitulate the essential features of clinical diabetic cardiomyopathy. The therapeutic effects of RSV prevented STZ- and HFD-induced myocardial oxidative stress and mitochondrial dysfunction and eventually rescued the impaired cardiac function and hypertrophy. Notably, diabetes- or high-glucose-induced oxidative stress and mitochondrial dysfunction in myocardial tissues or cardiomyocytes were mostly prevented by RSV through SIRT1-mediated PGC-1a deacetylation, thereby suggesting possible therapeutic applications in patients with diabetic cardiomyopathy.

In the present study, diabetes induced cardiac dysfunction and hypertrophy successfully, as shown by the increased heart weight-to-body weight ratio; decreased LVEF, LVFS, E/ A ratio and increased left ventricular chamber diameters. However, all cardiac injuries were attenuated by administration of RSV. A recent study revealed that sucrose feeding for 8 weeks induced insulin resistance that was not affected by cardiomyocyte cross-sectional area according to H\&E staining ${ }^{[37]}$; these controversial results may be attributed to the fact that the sucrose-enriched dietary model is in the early stages of development of type 2 diabetes and metabolic syndrome. Furthermore, cardiac hypertrophy was also confirmed by increased levels of pro-hypertrophic markers, such as ANP, BNP and $\beta-\mathrm{MHC}$, in cardiac tissues. Similarly, all of these parameters were decreased by RSV treatment. A direct role for RSV in remodeling is also supported by the observation that RSV attenuated high glucose-induced expression of ANP, BNP and $\beta$-MHC in cultured $\mathrm{H} 9 \mathrm{c} 2$ cells. These findings, consistent with those of a previous study ${ }^{[38,39]}$, demonstrate that RSV treatment might represent a realistic strategy to block the progression of cardiac dysfunction and hypertrophy.

The beneficial effects of RSV treatment were also reported to control hyperglycemia in short-term type 1 diabetes (2 weeks after STZ injection) rather than long-term type 1 diabetes, which may be related to its ability to induce anti-oxidant enzymes and its insulin-like activity. However, RSV treatment has been shown to prevent glucose-induced insulin secretion and improve insulin sensitivity in type 2 diabetes $^{[40,41]}$. Indeed, in the present study, RSV treatment for 16 weeks improved insulin sensitivity to some extent as shown by decreases in fasting blood glucose, $\mathrm{HbA1c}$, and serum TG and TC. However, these levels did not reach the normal range. That is to say, all diabetic rats after RSV treatment were still in the diabetic range, although the data in Figure 1 indicate that RSV treatment blocked diabetes-induced cardiac dysfunction and hypertrophy. These data indicate that the cardioprotective effects of RSV are not completely dependent on improved hyperglycemia and dyslipidemia. Furthermore, disorders of glucose and lipid metabolism are known to be closely related to impaired mitochondrial function. Interestingly, we found impaired cardiac mitochondrial function in our present study as shown by decreased ATP content, mtDNA content, PGC-1a and NRF expression, as well as increased oxidative stress. These results indicate that lowered blood glucose is more likely a consequence of mitochondrial function improvement after RSV administration rather than the hypothesis that lowered blood glucose is the cause of cardiac function improvement.

The roles of mitochondrial dysfunction in regulating glucolipid metabolism and mediating cardiac dysfunction in diabetes have become increasingly evident. Recent studies have suggested that mitochondria are major sites of intracellular ROS production, and mitochondrial dysfunction has been considered the chief culprit in triggering cytosolic oxidative stress and cell death ${ }^{[42]}$. A previous report demonstrated that incubation under high-glucose conditions increased mitochondrial superoxide generation in cultured cardiomyocytes. Mitochondrial oxidative stress and ROS generation were also induced in both type- 1 and type- 2 diabetic mouse hearts, contributed to myocardial hypertrophy and dysfunction and were prevented by the mitochondria-targeted antioxidant mito-TEMPO ${ }^{[43]}$. Overexpression of catalase reduced $\mathrm{H}_{2} \mathrm{O}_{2}$ levels at least threefold in mice fed a high-fat diet, which was concomitant with rescued cardiac function ${ }^{[44]}$. Consistent with these results, we observed significantly increased levels of lipid peroxidative products, such as MDA, and reduced SOD activity both in diabetic hearts and high-glucose-cultured cardiomyocytes. Moreover, we found severely impaired mitochondrial dysfunction as evidenced by reduced ATP content, mtDNA content, and PGC-1a and NRF expression, which indicated that cardiac oxidative damage and mitochondrial injury occurred in the diabetic model. In addition, we observed significantly elevated levels of UCP2 mRNA and protein expression both in type 2 diabetic rats and cardiomyocytes in accordance with a study that reported that UCP2 can be activated by superoxide anion $\left(\mathrm{O}_{2}{ }^{-}\right)$as a feedback mechanism ${ }^{[45]}$. However, some conflicting evidence has also been observed in previous studies showing that UCP2 is associated with reduced oxidative stress and protection of cardiovascular function ${ }^{[36]}$. Thus, it would be interesting to evaluate the effect of UCP2 in a model of diabetic cardiomyopathy; however, this experiment is beyond the scope of this study. RSV appears to counter mitochondrial ROS production by inducing antioxidant gene expression, thereby maintaining the cytosolic redox states and exhibiting cardioprotective effects $^{[46]}$. Das et al found that dietary intake of RSV prevents the progression of iron overload-induced injury and cardiomyopathy by correcting oxidative stress and myocardial fibrosis as shown by reduced SOD1 and glutathione levels and increased levels of myocardial disease markers ${ }^{[47]}$. Resveratrol also ameliorates myocardial fibrosis by inhibiting the ROS/ERK/TGF- $\beta$ / periostin pathway in STZ-induced diabetic mice ${ }^{[12]}$. In our present study, RSV treatment suppressed lipid peroxidation and increased cardiac SOD contents, thus rescuing mitochondrial function and restoring normal UCP2 expression. These results illustrate a key anti-oxidant effect of RSV against diabetes-induced oxidative stress in type 2 diabetic myocardial tissues. Meanwhile, a direct role for SIRT1 in oxidative stress and mitochondrial dysfunction is supported 
by the observation that a SIRT1 inhibitor or si-SIRT1 suppressed diabetes- or high-glucose-induced downregulation of SOD activity, ATP and COX 1 contents and PGC-1a and NRF expression in cultured $\mathrm{H} 9 \mathrm{c} 2$ cells.

The SIRT1/PGC-1a signaling pathway is an important regulator of cardiac mitochondrial function in normal or pathological conditions, including diabetes and ischemia reperfusion. SIRT1 deacetylates a variety of proteins and regulates mitochondrial function, inflammatory responses and stress resistance ${ }^{[48,49]}$, and PGC-1a is considered a major downstream target of SIRT1 ${ }^{[50]}$, which is responsible for regulating multiple genes involved in oxidative phosphorylation and mitochondrial function ${ }^{[51]}$. Although Lagouge et al showed that RSV predominantly relies on SIRT1 activation to achieve its effects on PGC-1a-dependent gene expression, these conclusions were all based on muscle, brown adipose tissues and $\mathrm{C} 2 \mathrm{C} 12$ cells, not cardiomyocytes or hearts, let alone a diabetic cardiomyopathy model ${ }^{[40]}$. SIRT3 was also reported to be a crucial deacetylase that regulates PGC-1a to exert cardioprotective effects in insulin resistance-induced cardiac injury ${ }^{[37,52]}$, while RSV possesses the unique ability to activate SIRT1 to regulate anti-oxidant and metabolic properties ${ }^{[12,47]}$. Thus, although previous studies have focused on the SIRT1-PGC-1a pathway, it is unclear whether RSV-ameliorated diabetic cardiomyopathy is dependent on the SIRT1-PGC-1a pathway; additionally, how RSV regulates PGC-1a and whether SIRT1 is required for RSV to regulate PGC-1a are unknown. In the present study, our in vivo and in vitro experiments, which both showed low levels of SIRT1, exhibited prompt hypertrophic responses and PGC-1a acetylation, whereas RSV administration reversed these phenomena. Additionally, treatment with the SIRT1 inhibitor sirtinol or si-SIRT1 abolished the effects of RSV and induced hyperacetylation of PGC-1a and cardiac dysfunction. These results provided direct evidence that RSV therapy activated SIRT1 to reduce acetylation of myocardial PGC-1a in response to high glucose and oxidative stress, supplying a key molecular basis for RSV therapeutic action. Taken together, the innovations of this study are as follows: (1) RSV prevents diabetic cardiomyopathy through the SIRT1-PGC-1a pathway. (2) RSV regulates PGC-1a by deacetylation. (3) SIRT1 is necessary for RSV to deacetylate PGC-1a.

\section{Conclusion}

In summary, these results provide compelling evidence for RSV protection during diabetic cardiac dysfunction by ameliorating oxidative stress and mitochondrial dysfunction and supply a deeper understanding of the regulatory role of SIRT1 on PGC-1a deacetylation in diabetic heart mitochondrial dysfunction. These results confirm that the SIRT1/PGC-1a pathway is a therapeutic target for treating diabetic cardiomyopathy.

\section{Acknowledgements}

This study was supported by the Program for the New Xiangya Talent Projects of the Third Xiangya Hospital of Central South University (№ JY201605).

\section{Author contribution}

Wei-jin FANG and Shi-kun LIU designed research; Wei-jin FANG, Chun-jiang WANG and Yang HE performed research; Yu-lu ZHOU and Xiang-dong PENG contributed new analytical tools and reagents; Wei-jin FANG analyzed data; Wei-jin FANG wrote the paper.

\section{References}

1 Beck-Nielsen H, Solomon TP, Lauridsen J, Karstoft K, Pedersen BK, Johnsen SP, et al. The danish centre for strategic research in type 2 diabetes (DD2) study: expected outcome from the DD2 project and two intervention studies. Clin Epidemiol 2012; 4 (Suppl 1): 21-6.

2 Tribouilloy C, Rusinaru D, Mahjoub H, Tartiere JM, Kesri-Tartiere L, Godard S, et al. Prognostic impact of diabetes mellitus in patients with heart failure and preserved ejection fraction: a prospective fiveyear study. Heart 2008; 94: 1450-5.

3 Giacco F, Brownlee M. Oxidative stress and diabetic complications. Circ Res 2010; 107: 1058-70.

4 Lin MT, Beal MF. Mitochondrial dysfunction and oxidative stress in neurodegenerative diseases. Nature 2006; 443: 787-95.

5 Bombicino SS, Iglesias DE, Mikusic IA, D'Annunzio V, Gelpi RJ, Boveris $A$, et al. Diabetes impairs heart mitochondrial function without changes in resting cardiac performance. Int J Biochem Cell Biol 2016; 81: 335-45.

6 Bagul PK, Deepthi N, Sultana R, Banerjee SK. Resveratrol ameliorates cardiac oxidative stress in diabetes through deacetylation of NFkB-p65 and histone 3. J Nutr Biochem 2015; 26: 1298-307.

7 Veeranki S, Givvimani S, Kundu S, Metreveli N, Pushpakumar S, Tyagi SC. Moderate intensity exercise prevents diabetic cardiomyopathy associated contractile dysfunction through restoration of mitochondrial function and connexin 43 levels in $d b / d b$ mice. J Mol Cell Cardiol 2016; 92: 163-73.

8 Hung LM, Chen JK, Huang SS, Lee RS, Su MJ. Cardioprotective effect of resveratrol, a natural antioxidant derived from grapes. Cardiovasc Res 2000; 47: 549-55.

9 Ignatowicz E, Baer-Dubowska W. Resveratrol, a natural chemopreventive agent against degenerative diseases. Pol J Pharmacol 2001; 53: 557-69.

10 Zhou X, Chen M, Zeng X, Yang J, Deng H, Yi L, et al. Resveratrol regulates mitochondrial reactive oxygen species homeostasis through Sirt3 signaling pathway in human vascular endothelial cells. Cell Death Dis 2014; 5: e1576.

11 Guo S, Yao Q, Ke Z, Chen H, Wu J, Liu C. Resveratrol attenuates high glucose-induced oxidative stress and cardiomyocyte apoptosis through AMPK. Mol Cell Endocrinol 2015; 412: 85-94.

12 Wu H, Li GN, Xie J, Li R, Chen QH, Chen JZ, et al. Resveratrol ameliorates myocardial fibrosis by inhibiting ROS/ERK/TGF-beta/ periostin pathway in STZ-induced diabetic mice. BMC Cardiovasc Disord 2016; 16: 5.

13 Mohammadshahi M, Haidari F, Soufi FG. Chronic resveratrol administration improves diabetic cardiomyopathy in part by reducing oxidative stress. Cardiol J 2014; 21: 39-46.

14 Guo R, Liu W, Liu B, Zhang B, Li W, Xu Y. SIRT1 suppresses cardiomyocyte apoptosis in diabetic cardiomyopathy: an insight into endoplasmic reticulum stress response mechanism. Int J Cardiol 2015; 191: 36-45.

15 Reed MJ, Meszaros K, Entes LJ, Claypool MD, Pinkett JG, Gadbois TM, et al. A new rat model of type 2 diabetes: the fat-fed, streptozotocintreated rat. Metabolism 2000; 49: 1390-4.

16 Liang D, Zhong P, Hu J, Lin F, Qian Y, Xu Z, et al. EGFR inhibition protects cardiac damage and remodeling through attenuating oxidative stress in STZ-induced diabetic mouse model. J Mol Cell Cardiol 2015; 82: 63-74.

17 Cong W, Ruan D, Xuan Y, Niu C, Tao Y, Wang Y, et al. Cardiac-specific 
overexpression of catalase prevents diabetes-induced pathological changes by inhibiting NF-kappaB signaling activation in the heart. J Mol Cell Cardiol 2015; 89 (Pt B): 314-25.

18 Wang B, Yang Q, Sun YY, Xing YF, Wang YB, Lu XT, et al. Resveratrolenhanced autophagic flux ameliorates myocardial oxidative stress injury in diabetic mice. J Cell Mol Med 2014; 18: 1599-611.

19 Li C, Wang Y, Qiu Q, Shi T, Wu Y, Han J, et al. Qishenyiqi protects ligation-induced left ventricular remodeling by attenuating inflammation and fibrosis via STAT3 and NF-kappaB signaling pathway. PLoS One 2014; 9: e104255.

20 Wiesen K, Kaiser E, Schroder L, Scholz A, Ruppenthal S, Reil JC, et al. Cardiac remodeling in Galphaq and Galpha11 knockout mice. Int J Cardiol 2016; 202: 836-45.

21 Collins KA, Korcarz CE, Shroff SG, Bednarz JE, Fentzke RC, Lin H, et al. Accuracy of echocardiographic estimates of left ventricular mass in mice. Am J Physiol Heart Circ Physiol 2001; 280: H1954-62

22 Battiprolu PK, Hojayev B, Jiang N, Wang ZV, Luo X, Iglewski M, et al. Metabolic stress-induced activation of FoxO1 triggers diabetic cardiomyopathy in mice. J Clin Invest 2012; 122: 1109-18.

23 Han SS, Wang G, Jin Y, Ma ZL, Jia WJ, Wu X, et al. Investigating the mechanism of hyperglycemia-induced fetal cardiac hypertrophy. PLoS One 2015; 10: e139141

24 Kordas KS, Sperlagh B, Tihanyi T, Topa L, Steward MC, Varga G, et al. ATP and ATPase secretion by exocrine pancreas in rat, guinea pig, and human. Pancreas 2004; 29: 53-60.

25 Yu P, Zhang J, Yu S, Luo Z, Hua F, Yuan L, et al. Protective effect of sevoflurane postconditioning against cardiac ischemia/reperfusion injury via ameliorating mitochondrial impairment, oxidative atress and rescuing autophagic clearance. PLoS One 2015; 10: e134666.

26 Kuang J, Sun Y, Wang W, Ke H, Ye H. Myocardial apoptosis and injury of donor hearts kept in completely beating status with normothermic blood perfusion for transplants. Int J Clin Exp Med 2015; 8: 5767-73.

27 Yoshioka J, Chutkow WA, Lee S, Kim JB, Yan J, Tian R, et al. Deletion of thioredoxin-interacting protein in mice impairs mitochondrial function but protects the myocardium from ischemia-reperfusion injury. J Clin Invest 2012; 122: 267-79.

28 Zhang X, Zuo X, Yang B, Li Z, Xue Y, Zhou Y, et al. MicroRNA directly enhances mitochondrial translation during muscle differentiation. Cell 2014; 158: 607-19.

29 Yang X, Yang S, Chai H, Yang Z, Lee RJ, Liao W, et al. A novel isoquinoline derivative anticancer agent and its targeted delivery to tumor cells using transferrin-conjugated liposomes. PLoS One 2015; 10: e136649.

30 Xiong XX, Liu JM, Qiu XY, Pan F, Yu SB, Chen XQ. Piperlongumine induces apoptotic and autophagic death of the primary myeloid leukemia cells from patients via activation of ROS-p38/JNK pathways. Acta Pharmacol Sin 2015; 36: 362-74.

$31 \mathrm{Kim}$ HS, Woo JS, Joo HJ, Moon WK. Cardiac transcription factor Nkx2.5 is downregulated under excessive O-GIcNAcylation condition. PLoS One 2012; 7: e38053.

32 Suganuma I, Mori T, Ito F, Tanaka Y, Sasaki A, Matsuo S, et al. Peroxisome proliferator-activated receptor gamma, coactivator 1alpha enhances local estrogen biosynthesis by stimulating aromatase activity in endometriosis. J Clin Endocrinol Metab 2014; 99: E11918.

33 Wang WK, Wang B, Lu QH, Zhang W, Qin WD, Liu XJ, et al. Inhibition of high-mobility group box 1 improves myocardial fibrosis and dysfunction in diabetic cardiomyopathy. Int J Cardiol 2014; 172 : 202-12.

34 Kuwabara Y, Horie T, Baba O, Watanabe S, Nishiga M, Usami S, et al. MicroRNA-451 exacerbates lipotoxicity in cardiac myocytes and highfat diet-induced cardiac hypertrophy in mice through suppression of the LKB1/AMPK pathway. Circ Res 2015; 116: 279-88.

35 Mattiasson G, Sullivan PG. The emerging functions of UCP2 in health, disease, and therapeutics. Antioxid Redox Signal 2006; 8: 1-38.

36 Moukdar F, Robidoux J, Lyght O, Pi J, Daniel KW, Collins S. Reduced antioxidant capacity and diet-induced atherosclerosis in uncoupling protein-2-deficient mice. J Lipid Res 2009; 50: 59-70.

$37 \mathrm{Hu} \mathrm{N}$, Ren J, Zhang Y. Mitochondrial aldehyde dehydrogenase obliterates insulin resistance-induced cardiac dysfunction through deacetylation of PGC-1alpha. Oncotarget 2016; 7: 76398-414.

38 Yang Q, Wang HC, Liu Y, Gao C, Sun L, Tao L. Resveratrol cardioprotection against myocardial ischemia/reperfusion injury involves upregulation of adiponectin levels and multimerization in type 2 diabetic mice. J Cardiovasc Pharmacol 2016; 68: 304-12.

39 Bresciani L, Calani L, Bocchi L, Delucchi F, Savi M, Ray S, et al. Bioaccumulation of resveratrol metabolites in myocardial tissue is dose-time dependent and related to cardiac hemodynamics in diabetic rats. Nutr Metab Cardiovasc Dis 2014; 24: 408-15.

40 Lagouge M, Argmann C, Gerhart-Hines Z, Meziane H, Lerin C, Daussin $\mathrm{F}$, et al. Resveratrol improves mitochondrial function and protects against metabolic disease by activating SIRT1 and PGC-1alpha. Cell 2006; 127: 1109-22.

41 Szkudelski T. Resveratrol inhibits insulin secretion from rat pancreatic islets. Eur J Pharmacol 2006; 552: 176-81.

42 Maharjan S, Oku M, Tsuda M, Hoseki J, Sakai Y. Mitochondrial impairment triggers cytosolic oxidative stress and cell death following proteasome inhibition. Sci Rep 2014; 4: 5896.

43 Ni R, Cao T, Xiong S, Ma J, Fan GC, Lacefield JC, et al. Therapeutic inhibition of mitochondrial reactive oxygen species with mito-TEMPO reduces diabetic cardiomyopathy. Free Radic Biol Med 2016; 90 : 12-23.

44 Sverdlov AL, Elezaby A, Qin F, Behring JB, Luptak I, Calamaras TD, et al. Mitochondrial reactive oxygen species mediate cardiac structural, functional, and mitochondrial consequences of diet-induced metabolic heart disease. J Am Heart Assoc 2016; 5: pii: e002555.

45 Echtay KS, Roussel D, St-Pierre J, Jekabsons MB, Cadenas S, Stuart $\mathrm{JA}$, et al. Superoxide activates mitochondrial uncoupling proteins. Nature 2002; 415: 96-9.

46 Ruan Y, Dong C, Patel J, Duan C, Wang X, Wu X, et al. SIRT1 suppresses doxorubicin-induced cardiotoxicity by regulating the oxidative stress and p38MAPK pathways. Cell Physiol Biochem 2015; 35 : 1116-24.

47 Das SK, Wang W, Zhabyeyev P, Basu R, McLean B, Fan D, et al. Ironoverload injury and cardiomyopathy in acquired and genetic models is attenuated by resveratrol therapy. Sci Rep 2015; 5: 18132.

48 Milne JC, Lambert PD, Schenk S, Carney DP, Smith JJ, Gagne DJ, et al. Small molecule activators of SIRT1 as therapeutics for the treatment of type 2 diabetes. Nature 2007; 450: 712-6.

49 Lavu S, Boss O, Elliott PJ, Lambert PD. Sirtuins-novel therapeutic targets to treat age-associated diseases. Nat Rev Drug Discov 2008; 7 : 841-53.

50 Gallardo-Montejano VI, Saxena G, Kusminski CM, Yang C, McAfee JL, Hahner L, et al. Nuclear Perilipin 5 integrates lipid droplet lipolysis with PGC-1alpha/SIRT1-dependent transcriptional regulation of mitochondrial function. Nat Commun 2016; 7: 12723.

51 Mootha VK, Lindgren CM, Eriksson KF, Subramanian A, Sihag S, Lehar J, et al. PGC-1alpha-responsive genes involved in oxidative phosphorylation are coordinately downregulated in human diabetes. Nat Genet 2003; 34: 267-73.

52 Klaman LD, Boss O, Peroni OD, Kim JK, Martino JL, Zabolotny JM, et al. Increased energy expenditure, decreased adiposity, and tissuespecific insulin sensitivity in protein-tyrosine phosphatase 1B-deficient mice. Mol Cell Biol 2000; 20: 5479-89. 